\title{
Robust Portfolio Allocation for a Bank under Inflation
}

\author{
Ryle S. Perera \\ Department of Applied Finance, Faculty of Business and Economics, Macquarie University, Sydney, Australia \\ Email: ryle.perera@mq.edu.au
}

How to cite this paper: Perera, R.S. (2018)

Robust Portfolio Allocation for a Bank under Inflation. Theoretical Economics Letters, 8, 3361-3394.

https://doi.org/10.4236/tel.2018.815207

Received: September 28, 2018

Accepted: November 23, 2018

Published: November 26, 2018

Copyright $\odot 2018$ by author and Scientific Research Publishing Inc. This work is licensed under the Creative Commons Attribution International License (CC BY 4.0).

http://creativecommons.org/licenses/by/4.0/

\begin{abstract}
In this study, we develop a robust portfolio allocation model for a bank in an incomplete market with inflation (a non-tradeable stochastic factor). The optimality criterion of the investments is established on a functional via a modified version of the monotone mean-variance preferences. An increase in anticipated inflation will increase the interest rate, while reducing the expected net stream of dollar receipts in the loan portfolio. Eventually whilst existing loans mature and are re-negotiated (at the higher interest rate), the interest rate is earned by the bank on existing loans are locked up. Under such explicit risk aggregation paradigm, we formulate this problem as a stochastic differential game (SDG) and apply the Hamilton-Jacobi-Bellman-Isaacs (HJBI)-equation to derive the optimal investment strategy. We discuss the dynamics of myopic optimal portfolio and the intertemporal hedging demand portfolio of the optimal portfolio holdings. We describe the dynamics of the total capital ratio under Basel III regulations. Finally, we show that our solution coincides with the solution to classical Markowitz optimization problem with risk aversion coefficient depends on stochastic factor. Our results confirm that the banker's optimal holdings and the trade-off between holding a myopically optimal portfolio and intertemporal hedging demand are determined by the derivatives of marginal utility with respect to the state variable.
\end{abstract}

\section{Keywords}

Basel III Capital Accord, Capital adequacy Ratio (CAR), HJBI Equation, Monotone Preferences, Inflation. Stochastic Differential Game

\section{Introduction}

The banking sector has been subject to constant changes in the economic environment over the past two decades. The Basel Committee on Banking Supervi- 
sion lays down regulations and supervises the behavior of the banking industry, by imposing minimal capital requirements and other measures. One of the deficiencies of the 1988 Basel Accord (Basel I) was its failure to distinguish between levels of credit risk in assets in general [1] [2]. According to Ferguson [3] this further increased the divergence between economic and regulatory capital requirements, making the capital adequacy ratio less reliable as a measure of the financial health of banking institutions. Theoretical evidence of the 1988 Accord suggested that the revised Basel Accord may influence the structure of a bank's balance sheet. Berger and Udell [4] examined the relationship between commercial loans and the risk-based capital requirements that operate as a regulatory tax. Jones [5] proposes regulatory capital arbitrage as an incentive to adjust on-and-off-balance sheet activities to the 1988 capital requirement. Since then numerous researchers [6] [7] [8] [9] [10] have carried out empirical studies in order to point out that the risk-based capital requirements caused a reduction in bank lending under Basel I. Studies by [9] [11] [12] [13] have examined the capital constraints on banks and response to the revised capital requirements. For a more comprehensive overview on Basel I and Basel II, we refer the reader to [14]-[22].

Basel II Capital Accord of June 2004 lays down regulations through more risk-sensitive minimum capital requirements. Basel III was agreed upon in 2010-11, and was scheduled to be introduced from 2013-2015. However, the date of implementation has been revised further to 31 March 2019. The third instalment of the Basel Accords was developed in response to the deficiencies in financial regulation revealed in the financial crisis of 2007-08. Basel III is intended to strengthen bank capital requirements by increasing bank liquidity and decreasing bank leverage. Unlike Basel I and Basel II, which focuses primarily on the level of bank loss reserves that banks are required to hold, Basel III focuses primarily on the risk of a run on the bank, requiring differing levels of reserves for different forms of bank deposits and other borrowings. Therefore, Basel III does not, for the most part, supersede the guidelines of Basel I and Basel II; rather it reinforces the intentions of the accord, see [23]. This provides incentives for greater awareness of differences in risk through more risk-sensitive minimum capital requirements based on numerical formulae. The Capital Adequacy Ratio (CAR) is a measure of the amount of a bank's capital relative to the amount of its credit exposures, see [16] [24] [25]. Therefore, maintaining minimum CAR is to guarantee that banks are prepared to absorb a reasonable level of loss before becoming insolvent and will help to promote the stability and effectiveness of the banking system.

On the other hand, the nominal interest rates quoted in financial markets are formed in the processes of contracting between borrowers and lenders and an increase in anticipated inflation raises the nominal interest rate. This increases the number of dollars that creditors or debtors who are transacting in nominal financial instruments except to receive or pay when a loan matures. If these expectations are realized, all nominal values will be higher at maturity. A banker's 
nominal assets and liabilities typically mature at different dates. At any given moment, the maturity dates of a bank's asset generally extend beyond those of its liabilities. In other words, interest rate change affects the payments stream obligated by the banker's liabilities before it affects the bank's receipt stream. Consequently, an increase in interest rate reduces the expected net stream of dollar receipts as the banker's creditors, renegotiate for a higher interest rate, while the interest rate earned by the bank on its existing loans eventually mature and are renegotiated at the higher interest rate, the interest rate earned by the bank on its existing loans is locked up. Of course, the loans eventually mature and are renegotiated at the higher nominal rate, but the banker's capital is reduced nonetheless. Banker's optimal asset portfolio decision requires that the expected returns from the two portfolios are equalized in equilibrium. Therefore, in the presence of anticipated inflation the interest rate at which banks lend to firms decreases the banks' net worth and show that a deterioration in banks' net worth or a strengthening of capital requirements may increase the interest rate at which banks lend to firms and hence dampen lending and output. As a result, the supply of capital to goods producing firms will fall, reducing output. As output declines, aggregate demand weakens and inflation declines. The initial effect, originating from the change in the maximum loss, brings about second-round effects on the macro-economy through endogenous developments in the banks' net worth. When the initial effect leads to a decline in the banks' net worth, it also dampens output and inflation through changes in the risk taking capacity originating from insufficiency. Hence, bank's capital has a direct effect on the upper bound on bank assets under Basel III capital requirement and thereby bank's lending ability. Importantly, there are two conditions required for the bank capital channel to operate. First, banks should have no excess capital that can be used to buffer against shocks that deplete bank capital. Secondly, the capital market is imperfect in that it is costly for a bank to raise capital

Literature regarding quadratic optimization dates back to Markowitz in the 1950's. In his mean-variance analysis the theory of combining risky assets to minimize the variance of return (i.e., risk) at any desired mean return is examined. The locus of optimal mean-variance combinations is called the efficient frontier, on which all rational investors desire to be positioned. Since then there has been continued interest to incorporate specific features to the Markowitz [26] model, such as the monotone characteristics. Maccheroni et al. [27] addressed this issue and formulated a new class of monotone preferences that coincide with mean-variance preferences on their domain of monotonicity, but differ where mean-variance preferences fail to be monotone. Moreover, they showed the functional associated with this new class of preferences details the monotone mean-variance preferences and its advantage over mean-variance preferences Maccheroni et al. [27]. In a dynamic optimization setting a modification of Maccheroni type objective function has been analyzed by [18] [28]-[33]. The problem of maximizing Maccheroni's 2009 functional is a max-min problem that naturally forms a stochastic differential game (SDG). In 
the literature there are two main approaches in determining the solution to such games either via the maximum principle or Backward Stochastic Differential Equations (BSDEs). The BSDE approach is based on the dynamic principle and Hamilton-Jacobi-Bellman equations (Hamilton-Jacobi-Bellman-Isaccs (HJBI) for differential games). In this study we use HJBI equation for the game and simplify to a linear form by applying some transformations. As a by-product we obtain a formula for the optimal strategy.

The novelty, of this study is the development of a robust portfolio allocation/management model for a bank with respect to inflation (non-tradable stochastic state variable) and provisional capital process that maximizes performance criterion of a modified version of the monotone mean-variance functional. Assuming that the banker can invest in treasuries, a stock index and a loan portfolio, we formulate the portfolio optimization problem as a SDG and it is solved via the HJBI equation to derive the optimal investment strategy. Optimality criterion is constructed via a functional by modifying the monotone mean-variance performance. We discuss the banker's portfolio compositional decision based on the expected returns from the two portfolio holdings subject to the equalization in equilibrium. We examine the dynamics of myopic optimal portfolio and the intertemporal hedging demand portfolio of the optimal portfolio holdings. We then derive the Basel III CAR. Compliance of minimum CAR is modeled under the assumption retained earnings, loan-loss reserves, the market and shareholder-bank owner relationship. In this study, Basel III CAR given by

$$
Q=\frac{C}{a_{r w}},
$$

where $C$ represents the total capital and $a_{r w}$ the total risk-weighted assets (TRWAs) of the bank, respectively.

Recalling Maccheroni et al. [27], we have (optimal asset portfolio decision requires that the expected returns from the two assets are equalized in equilibrium)

$$
X \rightarrow \inf _{Q \in \mathcal{Q}} \mathbb{E}^{Q}\left[X+\frac{1}{2 \theta} C(Q \mid P)\right], \theta>0,
$$

where $\theta$, is a risk aversion coefficient, $C(Q \mid P)$, called a penalty function, $\mathcal{Q}$ is a class of all probability measures, $P$ is a given probability measure and

$$
C(Q \mid P)= \begin{cases}\mathbb{E}^{P}\left[\left(\frac{\mathrm{d} Q}{\mathrm{~d} P}\right)^{2}\right]-1, & \text { if } Q \ll P, \\ +\infty, & \text { otherwise }\end{cases}
$$

where $C(Q \mid P)$ is known as the Gini concentration index. Due to technical difficulties, of Equation (1), we consider the set $\mathcal{Q}$ to be all absolutely continuous probability measures which have square intagrable Radon-Nikodym derivative of the form Equation (15). This modification of monotone mean-variance function is still monotone and the performance criterion of convex risk measure $\Lambda(X)$, is defined as: 


$$
\Lambda(X)=\sup _{Q \in \mathcal{Q}} \mathbb{E}^{Q}\left[-X-\frac{1}{2} \frac{\mathrm{d} Q}{\mathrm{~d} P}\right], \theta>0,
$$

satisfying the following three axioms:

1) Convexity: If $\alpha \in(0,1)$, then $\Lambda(\alpha X+(1-\alpha) Y) \leq \alpha \Lambda(X)+(1-\alpha) \Lambda(Y)$,

2) Monotonicity: if $X \leq Y$, then $\Lambda(Y) \leq \Lambda(X)$,

3) Translation invariance: $\beta \in \mathbb{R}$, then $\Lambda(X+\Upsilon)=\Lambda(X)-\Upsilon$.

Hence, the problem of maximizing Equation (1) becomes a max-min problem and naturally forms a stochastic differential game for optimization problem.

This article makes the following contributions to the literature:

1) Introducing banker's provision capital risk process as a controllable diffusion process, we extend [31] analysis into robust portfolio allocation/management framework with inflation. This allows the banker to deal with the issue of bank capital adequacy and risk management in an incomplete market within a stochastic dynamic setting. This empowers the banker to regulate his/her provision capital risk process by controlling the amount of wealth invested in the loan portfolio as well as the amount of wealth invested in the stock index. A negative/or positive correlation between the provision capital process and the capital loss/or gains from the loan portfolio is captured via $\hat{\rho}_{2}$. A negative/or positive correlation will capture the influence of provision capital risk process on the financial market holdings via $\hat{\rho}_{1}$. Consequently, we can show how a negative/positive correlation of $\hat{\rho}_{1}$, and $\hat{\rho}_{2}$ affect the banker's myopic demand and intertemporal hedging demands on optimal polices.

2) We argue that the relevance of inflation risk stems not only from banker's concerns with real return volatility, or interest lost but also from the fact that inflation is a proxy for the variation of the investment opportunity set.

3) We formulate this problem as a stochastic differential game and use HJBI equation to derive optimal investment strategy.

4) Optimality criterion of the optimal investment is based on a functional as a modification of a monotone mean-variance preference.

5) We provide a verification theorem and describe the dynamics of the total capital ratio under Basel III regulations

6) We show that our solution coincides with the solution to classical Markowitz problem where the risk aversion coefficient is dependent on stochastic factor.

7) Our results confirm that the presence of inflation risk alters the banker's optimal holdings and the trade-off between the myopically demand optimal portfolio and intertemporal hedging demand portfolio is determined by the derivatives of marginal utility with respect to the state variable.

The remainder of this paper is organized as follows: Section 2 and Section 3 contains the description of the investment opportunities, market setting and model set up and in Section 4 we formulate the bank's investment problem subject to banker's provision process as a Maccheroni type SDG between the banker and the market. We then derive the HJBI equation, formulate the verification theorem and transform our equation to linear form. This is followed by a com- 
parative analysis and discussion of the useful properties of this solution. In Section 5 we derive an explicit Indigo Partial Differential Equation (IPDE) for the dynamics of the Basel III CAR by dividing the total bank capital and TRWAs under provision capital process paradigm. In Section 6 we compare the result with solution to classical mean-variance optimization problem. Section 7 concludes the paper.

\section{Formulation of the Banking Model}

We consider a financial market which is continuously open over the fixed time interval $[0, T]$. We work within a filtered probability space $(\Omega, \mathcal{F}, P)$ with $\mathbb{F}=(\mathcal{F}(t))_{0 \leq t \leq T}$, where $P$ is the reference probability measure and $\Omega$ denotes the information structure. The mathematical model for a continuous-time market allows at least two types of financial assets (treasuries, and a stock index fund) to be bought and sold without incurring any transaction costs or restriction on short sales. Issuing of loans is considered to be a third investment opportunity for the bank. We assume that the expected rate of inflation is not observable, but must be inferred from observation of the price level itself and this inflationary risk in the market is captured by an external stochastic factor $O:=(O(t))_{0 \leq t \leq T}$, with $O(s)=o$. To capture the operation and management strategies of banks, we need to consider the balance sheet, which records the bank's assets (uses of funds) and bank's liabilities (source of funds). The items on the balance sheet behave in an unpredictable manner, due to the uncertain behavior of the activities related to the evolution of treasuries, loan demand, risky and riskless investments, deposits, loan repayments, borrowing and eligible capital. As in [24], we define the balance sheet of a commercial bank at time $t$ as

$$
M(t)+S(t)+L(t)=D(t)+B(t)+C(t),
$$

where, $M, S, L, D, B$ and $C$ are the treasuries, securities, loans, deposits, borrowing and bank capital, respectively. Each of these variables will be regarded as a function of $\Omega \times \mathbb{R}_{+} \rightarrow \mathbb{R}_{+}$.

\subsection{Treasures, Securities and Loans}

A bank reserve is the currency deposit that is not lent out to the bank's clients. A small fraction of the total deposits is held internally by the bank in cash vaults or deposited with the central bank. Minimum reserve requirements are established by central banks in order to ensure that the financial institutions will be able to provide clients with cash upon request. Bank reserves are typically held by financial institutions to avoid bank runs and have sufficient cash on hand, should an unexpected and large withdrawal request come up. Bank reserves are divided into required reserves and excess reserves. Because of the banking industry's importance to the economy, national authorities regulate banks by obligating them to hold a certain amount of required reserves with central banks. Excess reserves represent any vault cash that banks hold that is in excess of the required reserves amount. Banks typically have low incentive to maintain excess reserves 
because cash earns the rate of return of zero and can lose value over time due to inflation. Thus, under normal circumstances, banks minimize their excess reserves and lend out money to clients rather than holding cash in their vaults. Bank reserves decrease during periods of economic expansion and increase during recessions.

These funds are not used to lend to customers or to meet day-to-day currency withdrawals. Treasury securities and bonds are issued by the national treasury as a means of borrowing money to meet government expenditures that have not been covered by tax revenues. Marketable securities are stocks and bonds that can be swiftly converted into cash, hence are highly liquid assets. We suppose that a commercial bank raises funds to invest in a risky asset, in this case a loan. The interest rate on the loan is denoted by $r(t)$.

\subsection{Total Bank Capital}

Banks can raise their capital by selling new equity, retaining earnings, issuing debt or building up loan reserves. By nature, the dynamics of bank capital is stochastic due to uncertainty related to debt and shareholder contributions. However, in theory the bank can decide on the rate at which debt and equity is raised. According to Basel III, the bank capital can be portioned into so-called Tier 1 and Tier 2 capital, i.e., $C(t)=C_{T 1}(t)+C_{T 2}(t)$.

Tier 1 capital is the book value of its stocks, $E(t)$ plus retained earnings $E_{r}(t)$. Tier 2 capital (collectively known as supplementary capital) is the sum of subordinate debt, $S_{D}(t)$ and $R_{L}(t)$ loan-loss reserves. As a result, we have

$$
C_{r}(t)=E(t)+E_{r}(t),
$$

and

$$
C_{T 2}(t)=S_{D}(t)+R_{L}(t) .
$$

Therefore, the total bank capital can be expressed as

$$
C(t)=E(t)+E_{r}(t)+S_{D}(t)+R_{L}(t) .
$$

The market value of subordinate debt at time $t$ may be given by

$S_{D}(t)=S_{D}(0) \exp \left(\int_{0}^{t} r(u) \mathrm{d} u\right)$.

\subsection{Dynamics of Total Capital}

We assume that the bank holds capital in $n+1$ categories, $n$ of which are referred to as bank equity. Then the return on the ith bank equity is defined as

$$
d e_{i}(t)=e_{i}(t)\left[\left(r(t)+\sum_{j=1}^{n} \sigma_{i j} \eta_{j}\right) \mathrm{d} t+\sum_{j=1}^{n} \sigma_{i j} \mathrm{~d} \hat{W}_{j}(t)\right], i=1,2, \cdots, n,
$$

The co-variance matrix and the market price of risk are given by $\Psi=\left(\sigma_{i j}\right)_{i, j=1}^{n}$ and $\eta=\left(\eta_{1}, \cdots, \eta_{n}\right)^{\mathrm{T}}$, respectively and are assumed to be constants. $\mathrm{T}$, is the transpose of a vector or matrix. At time $t$ we assume that the bank capital is being converted into loan and marketable securities at the rate of $\rho_{X}(t)=\bar{\rho} X(t) \mathrm{d} t$ 
for a constant $\bar{\rho}$. Here, $X(t)$ represents the total asset portfolio of the bank.

Greenbaum \& Thakor [34] argues that excessive high capital requirements may result in banks taking on more risk and may lead to a bank acquiring higher levels of equity on order to become compliant. The upshot of such practices includes reduced liquidity and erosion of discipline in the bank's operation while defeating the purpose of the regulatory requirements. Therefore, capital requirement should be pitched at an appropriate level and banks should operate as near as possible to the minimum required level of capital. Therefore, it is essential to properly monitor and project the dynamics of the CAR. For this reason, in Section 6, we describe the dynamics of Basel III CAR as a SDE. Due to the non-dynamic character of retained earnings and loan-loss reserves, these aspects are not considered to be active constituents of bank capital. This implies $\mathrm{d} E_{r}(t)=\mathrm{d} R_{L}(t)=0, \forall t$. Hence the $C$-dynamics may be expressed as:

$$
\begin{aligned}
\mathrm{d} C(t) & =C(t) \sum_{i=1}^{n} w_{i} \frac{d e_{i}(t)}{e_{i}(t)}+\left(1-\sum_{i=1}^{n} w_{i}(t)\right) C(t) \frac{\mathrm{d} S_{D}(t)}{S_{D}(t)}-\bar{\rho} X(t) \mathrm{d} t \\
& =C(t)\left[\left(r(t)+w^{\mathrm{T}}(t) \Psi \eta\right) \mathrm{d} t+w^{\mathrm{T}}(t) \Psi \mathrm{d} \hat{W}(t)\right]-\bar{\rho} X(t) \mathrm{d} t,
\end{aligned}
$$

where $w^{\mathrm{T}}(t)$ are the proportions invested in securities. The diffusion term $w^{\mathrm{T}}(t) \Psi \mathrm{d} \hat{W}(t)$ in (9) establishes a correlation between bank capital and total risk-weighted assets.

\section{Financial Market Setting}

\subsection{Treasures}

The first of three assets, we consider treasury $M(t)$ which evolves according to

$$
\mathrm{d} M(t)=M(t) r(t) \mathrm{d} t, \quad M(0)=1,
$$

where $r(t)$ is the instantaneous nominal interest rate at time $t$ and is $\mathcal{F}_{t}$ measurable. Assume that $M(t)$ is a well-defined process.

\subsection{Stock Index Fund}

The evolution of the price process of the stock index fund $S:=(S(t))_{0 \leq t \leq T}$ is governed by the SDE:

$$
\left.\mathrm{d} S(t)=S(t)\left[\left(r(t)+\xi_{S}(O(t))\right) \mathrm{d} t+\sigma_{1}(O(t)) \mathrm{d} W_{1}(t)\right)\right], \quad S(0)=s>0,
$$

where $\xi_{S}(O(t))>0$, is the risk premia of the stock index, $\sigma_{1}(O(t))>0$, is positive volatility parameters that are continuous functions and assumed to satisfy all the required regularity conditions, in order to guarantee that the unique strong solution to Equation (11) exists. The unit market price of risk for the stock index is $\lambda_{1}(O(t))>0$ and the risk premium of the stock index fund is $\xi_{S}(O(t)):=\sigma_{1}(O(t)) \lambda_{1}(O(t))$.

\subsection{Loans}

Any loan is essentially an interest rate contingent claim and by Itô's lemma the dynamics of the loan price are assumed to follow according to the SDE 


$$
\mathrm{d} L(t)=L(t)\left[\left(r(t)+\xi_{L}(O(t))\right) \mathrm{d} t+\sigma_{L}(O(t)) \mathrm{d} W_{2}(t)\right] .
$$

$\xi_{L}(O(t))>0$ is the risk premia per loan in the loan portfolio, $\sigma_{L}(O(t))>0$, is the volatility parameter that are continuous functions and assumed to satisfy all the required regularity conditions, in order to guarantee that the unique strong solution to Equation (12) exists. We assume that the bank grants loans at a nominal interest rate or loan rate as a sum of instantaneous nominal interest rates, the market price of risk and the default risk premium. Here,

$\xi_{L}(O(t))=\tilde{\lambda}_{2}(O(t)) \sigma_{L}(O(t))+\varepsilon$, is the unit market price of risk for a loan. As in [35], the default risk premium $\varepsilon$, is the credit spread charged by the bank and it is the function of the probability of default $(P D)$, and the loss given default of the loan, $(L G D)($ Spread $=P D \times L G D)$. Main liabilities for the bank come from approving loans. Total outstanding number of loans at time $t$ is denoted by $L(t)$. Then the revenue from writing loan policies over the time period $(t, t+\mathrm{d} t)$ is given by $\xi_{L}(O(t)) w_{2}(t) \mathrm{d} t$. We also assume that bank can sell their loan portfolios to other banks.

\subsection{Inflation in the Economy}

We define the dynamics of the rate of inflation is given via the following SDE

$$
\mathrm{d} O(t)=a(O(t)) \mathrm{d} t+b(O(t))\left(\rho_{1} \mathrm{~d} W_{1}(t)+\rho_{2} \mathrm{~d} W_{2}(t)+\rho_{3} W_{3}(t)\right), O(0)=o,
$$

where the coefficients $a, b$ are continuous functions and satisfy all the required regularity conditions, in order to guarantee that the unique strong solution to Equation (13). $\left(\rho_{1}, \rho_{2}, \rho_{3}\right) \in[-1,1]$ are the correlation coefficients and $W_{1}, W_{2}$ and $W_{3}$ are three independent standard Brownian motion which are orthogonal to each other.

\subsection{Bank's Provision Capital Process}

The nominal interest rates quoted in financial markets are formed in the processes of contracting between borrows and lenders and an increase in anticipated inflation raises the nominal interest rate. This increases the number of dollars that creditors or debtors who are transacting in nominal financial instruments except to receive or pay when loan mature. If these expectations are realized, all nominal values will be higher at maturity. A banker's nominal assets and liabilities typically mature at different dates. At any given moment, the maturity dates of a bank's asset generally extend beyond those of its liabilities. In other words, interest rate change affects the payments stream obligated by the banker's liabilities before it affects the bank's receipt stream. Consequently, an increase in interest rate reduces the expected net stream of dollar receipts as the banker's creditors, renegotiate for a higher interest rate, while the interest rate earned by the bank on its existing loans eventually mature and are renegotiated at the higher interest rate, while the interest rate earned by the bank on its existing loans is locked up. Of course, the loans eventually mature and are renegotiated at the higher nominal rate, but the banker's capital is reduced nonetheless. We 
also argue that the relevance of inflation risk stem not only from banker's concerns with real return volatility but also from the fact that inflation is a proxy for the variation of the investment opportunity set. We define the bank's provision capital risk process as

$$
\mathrm{d} \pi(t)=v \mathrm{~d} t+\beta \mathrm{d} \tilde{W}(t),
$$

where $v$ and $\beta$ are two positive constants. $\tilde{W}(t)$ is another standard Brownian motion defined on the given filtered probability space and a negative/or positive correlation will capture the influence of provision capital risk process of the loan portfolio on the financial markets via $\hat{\rho}_{1}$. A negative/or positive correlation between the provision capital process and the capital loss/or gains from the loan portfolio via $\hat{\rho}_{2}$. Inflationary risk in the economy is captured via $\hat{\rho}_{3}$. We suppose that under $P$, there is a 4-dimensional Brownian motion such that the correlation coefficients are $\hat{\rho}_{1}, \hat{\rho}_{2}, \hat{\rho}_{3}$ and $\hat{\rho}_{4}$ respectively, with respect to $\tilde{W}$. Then $\tilde{W}(t)$ can be written as

$$
\tilde{W}(t)=\hat{\rho}_{1} W_{1}(t)+\hat{\rho}_{2} W_{2}(t)+\hat{\rho}_{3} W_{3}(t)+\hat{\rho}_{4} W_{4}(t)
$$

where $W_{4}(t)$ is another standard Brownian motion orthogonal to $W_{1}(t), W_{2}(t), W_{3}(t)$, we define $\hat{\rho}_{4}=\sqrt{1-\left(\hat{\rho}_{1}^{2}+\hat{\rho}_{2}^{2}+\hat{\rho}_{3}^{2}\right)}$ and $\left(\hat{\rho}_{1}, \hat{\rho}_{2}, \hat{\rho}_{3}, \hat{\rho}_{4}\right) \in[-1,1]$.

We assume that the probability measure is not precisely known and the banker knows only a class of possible measures. To construct banker's objective function, following [29], we consider the class of $\eta:=\left(\eta_{1}, \eta_{2}, \eta_{3}, \eta_{4}\right) \in \mathcal{M}$. as

$$
\begin{aligned}
\mathcal{Q}:= & \left\{Q \sim P \mid \frac{d Q}{d P}=\mathcal{E}\left(\int \eta_{1}(t) \mathrm{d} W_{11}(t)+\eta_{2}(t) \mathrm{d} W_{2}(t)\right.\right. \\
& \left.\left.+\eta_{3}(t) \mathrm{d} W_{3}(t)+\eta_{4}(t) \mathrm{d} W_{4}(t)\right)_{T}\right\},
\end{aligned}
$$

where $\mathcal{E}(\cdot)_{t}$ denotes the Doleans-Dade exponential and $\mathcal{M}$ denotes the set of all progressively measurable processes $\eta:=\left(\eta_{1}, \eta_{2}, \eta_{3}, \eta_{4}\right)$ such that $E\left(\frac{\mathrm{d} Q^{\eta}}{\mathrm{d} P}\right)^{2}<+\infty$ and $E\left(\frac{\mathrm{d} Q^{\eta}}{\mathrm{d} P}\right)=1 . Q^{\eta}$ denotes the measure determined by $\eta \in \mathcal{M}$. This implies that we have an additional family of stochastic processes $\left(Y^{\eta}(t), 0 \leq t \leq T\right)$, are given by the SDE

$$
\begin{aligned}
\mathrm{d} Y^{\eta}(t)= & \eta_{1}(t) Y^{\eta}(t) \mathrm{d} W_{1}(t)+\eta_{2}(t) Y^{\eta}(t) \mathrm{d} W_{2}(t)+\eta_{3}(t) Y^{\eta}(t) \mathrm{d} W_{3}(t) \\
& +\eta_{4}(t) Y^{\eta}(t) \mathrm{d} W_{4}(t), Y^{\eta}(s)=y .
\end{aligned}
$$

Moreover, note that $Y^{\eta}(T)=y \frac{\mathrm{d} Q^{\eta}}{\mathrm{d} P}$.

At time $t$, the banker chooses $w_{1}(t)$ amount to be invested in the risky stock index fund and $w_{2}(t)$ amount of outstanding loans included in the loan portfolio. Then the trading strategy is a pair of stochastic processes $\kappa(t):=\left(w_{1}(t), w_{2}(t)\right)$. Corresponding to a strategy $\kappa(t)$ the dynamics of the bank's portfolio under inflational risk control strategy can be written in an ex- 
plicit manner subject to an initial wealth $x$ as

$$
\begin{aligned}
& \mathrm{d} X^{(x, \kappa)}(t) \\
& =\left(1-w_{1}(t)-w_{2}(t)\right) \frac{\mathrm{d} M(t)}{M(t)}+w_{1}(t) \frac{\mathrm{d} S(t)}{S(t)}+w_{2}(t) \frac{\mathrm{d} L(t)}{L(t)}-w_{2}(t) \mathrm{d} \pi(t) \\
& =\left[r(t) X^{x, \kappa}(t)+\xi_{S}(O(t)) w_{1}(t)+\xi_{L}(O(t)) w_{2}(t)-v w_{2}(t)\right] \mathrm{d} t \\
& +\sigma_{1}(O(t)) w_{1}(t) \mathrm{d} W_{1}(t)+\sigma_{L}(O(t)) w_{2}(t) \mathrm{d} W_{2}(t)-w_{2}(t) \beta \mathrm{d} \tilde{W}(t),
\end{aligned}
$$

where $\xi_{S}(O(t))=\mu_{S}(O(t)-r(t)), \xi_{L}(O(t))=\mu_{L}(O(t)-r(t)) \cdot \mu_{S}(O(t))$ and $\mu_{L}(O(t))$ are the appreciation rates for the stock index and for a loan, respectively. We rewrite Equation (17) as:

$$
\begin{aligned}
\mathrm{d} X^{x, \kappa}(t)= & {\left[r(t) X^{x, \kappa}(t)+\xi_{S}(O(t)) w_{1}(t)+\xi_{L}(O(t)) w_{2}(t)-v w_{2}(t)\right] \mathrm{d} t } \\
& +\left(\sigma_{1}(O(t)) w_{1}(t)-\beta \hat{\rho}_{1} w_{2}(t)\right) \mathrm{d} W_{1}(t) \\
& +w_{2}(t)\left(\sigma_{L}(O(t))-\beta \hat{\rho}_{2}\right) \mathrm{d} W_{2}(t) \\
& -w_{2}(t) \beta \hat{\rho}_{3} \mathrm{~d} W_{3}(t)-w_{2}(t) \beta \hat{\rho}_{4} \mathrm{~d} W_{4}(t) \\
X(0)=x . &
\end{aligned}
$$

Definition 1. The control strategy $\kappa=\left(w_{1}(s), w_{2}(s)\right) ; t \leq s \leq T$ in the time interval $[t, T]$, is admissible if it satisfies the following assumptions:

1) $w_{1}, w_{2}$ are progressively measurable;

2) There exists an unique solution to Equation (14) and

$E_{x, t}^{\eta}\left[\sup _{t \leq s \leq T}\left|X_{s}^{\left(w_{1}, w_{2}\right)}\right|\right]<+\infty$ for all $\eta \in \mathcal{M}$, where $E^{\eta}$ denotes the expectation with respect to measure $Q^{\eta}$.

\section{Banker's Asset Optimization Strategy}

Shareholders of a bank expect a decent return on their capital investment. In order to maximize shareholder wealth, the bank management must strategically allocate the shareholder's wealth in investment strategies while minimizing the risks. (Shareholders will describe the uncertainty of an investment's success or failure as risk, and the bank management must monitor this risk because it affects the bank's cost of capital, market value and ultimately shareholder wealth). Consequently, changes in the bank's asset value are reflected in changes in the shareholder's equity. The value of the bank portfolio depends on the credit quality of borrowers (creditors) that the bank has lent money to conclude that the uncertainty in borrowers' future credit quality leads to uncertainty in the bank's future portfolio value. In order to formulate the banker's optimization problem as a Maccheroni type, we define the banker's objective as

$$
\begin{gathered}
J^{(\kappa, \eta)}(x, y, o, t):=E_{x, y, \delta, t}^{\eta}\left[-X^{(\kappa)}(T)-Y^{\eta}(T)\right] . \text { Then the banker's aim is to } \\
\qquad \operatorname{minsup}_{\eta \in \mathcal{M}} J^{(\kappa, \eta)}(x, y, o, t),
\end{gathered}
$$

over a class of admissible strategies $\mathcal{A}_{t}$.

Using [36] stochastic differential game we model problem (19) as a zero-sum 
stochastic differential game. We claim the measure $Q^{\eta}$ is controlled by the market (player 1) and the portfolio $\kappa$ is controlled by the banker (player 2). Under this setting we are looking for a saddle point $\left\{\left(w_{1}^{*}, w_{2}^{*}\right), \eta^{*}\right\} \in \mathcal{A}_{t} \times \mathcal{M}$ and the value function $V(x, y, o, t)$ such that

$$
J^{\left(\left(w_{1}^{*}, w_{2}^{*}\right), \eta\right)}(x, y, o, t) \leq J^{\left(\left(w_{1}^{*}, w_{2}^{*}\right), \eta^{*}\right)}(x, y, o, t) \leq J^{\left(\left(w_{1}, w_{2}\right), \eta^{*}\right)}(x, y, o, t)
$$

and $V(x, y, o, t)=J^{\left(\left(w_{1}^{*}, w_{2}^{*}\right), \eta^{*}\right)}(x, y, o, t)$.

\subsection{HJBI Equation and the Verification Theorem}

To establish a link between HJBI-equation and a saddle point to our problem in this subsection, we recall Equations (18), (16) \& (13) and setting $\mathcal{M}(t)=1$ for all $t$. i.e.

$$
\left\{\begin{aligned}
\mathrm{d} X^{\left(w_{1}, w_{2}\right)}(t)= & {\left[\xi_{S}(O(t)) w_{1}(t)+\xi_{L}(O(t)) w_{2}(t)-v w_{2}(t)\right] \mathrm{d} t } \\
& +\sigma_{1}(O(t)) w_{1}(t) \mathrm{d} W_{1}(t)+\sigma_{L}(O(t)) w_{2}(t) \mathrm{d} W_{2}(t) \\
& -w_{2}(t) \beta \mathrm{d} \tilde{W}(t), \\
\mathrm{d} Y^{\eta}(t)= & \eta_{1}(t) Y^{\eta}(t) \mathrm{d} W^{1}(t)+\eta_{2}(t) Y^{\eta}(t) \mathrm{d} W_{2}(t) \\
& +\eta_{3}(t) Y^{\eta}(t) \mathrm{d} W_{3}(t)+\eta_{4}(t) Y^{\eta}(t) \mathrm{d} W_{4}(t), \\
\mathrm{d} O(t)= & a(O(t)) \mathrm{d} t+b(O(t))\left(\rho_{1} \mathrm{~d} W_{1}(t)+\rho_{2} \mathrm{~d} W_{2}(t)+\rho_{3} W_{3}(t)\right) .
\end{aligned}\right.
$$

After applying the Girsanov transformation, we define the $Q^{\eta}$ dynamics of the system (20) as:

$$
\left\{\begin{aligned}
\mathrm{d} X^{\left(w_{1}, w_{2}\right)}(t)= & {\left[w_{1}(t) \xi_{S}(O(t))+w_{2}(t)\left(\xi_{L}(O(t))-v\right)+\left(w_{1}(t) \sigma_{1}(O(t))\right.\right.} \\
& \left.-\beta \hat{\rho}_{1} w_{2}(t)\right) \eta_{1}(t)+w_{2}(t)\left(\sigma_{L}(O(t))-\beta \hat{\rho}_{2}\right) \eta_{2}(t) \\
& \left.-w_{2}(t) \beta \hat{\rho}_{3} \eta_{3}(t)-w_{2}(t) \beta \hat{\rho}_{4} \eta_{4}(t)\right] \mathrm{d} t+\left(w_{1}(t) \sigma_{1}(O(t))\right. \\
& \left.-\beta \hat{\rho}_{1} w_{2}(t)\right) \mathrm{d} W_{1}(t)+w_{2}(t)\left(\sigma_{L}(O(t))-\beta \hat{\rho}_{2}\right) \mathrm{d} W_{2}(t) \\
& -w_{2}(t) \beta \hat{\rho}_{3} \mathrm{~d} W_{3}(t)-w_{2}(t) \beta \hat{\rho}_{4} \mathrm{~d} W_{4}(t), \\
\mathrm{d} Y^{\eta}(t)= & \left(\eta_{1}^{2}(t)+\eta_{2}^{2}(t)+\eta_{3}^{2}(t)+\eta_{4}^{2}(t)\right) Y^{\eta}(t) \mathrm{d} t+\eta_{1}(t) Y^{\eta}(t) \mathrm{d} W_{\eta_{1}}(t) \\
& +\eta_{2}(t) Y^{\eta}(t) \mathrm{d} W_{\eta_{2}}(t)+\eta_{3}(t) Y^{\eta}(t) \mathrm{d} W_{\eta_{3}}(t)+\eta_{4}(t) Y^{\eta}(t) \mathrm{d} W_{\eta_{4}}(t), \\
\mathrm{d} O(t)= & \left(a(O(t))+b(O(t)) \rho_{1} \eta_{1}(t)+b(O(t)) \rho_{2} \eta_{2}(t)+b(O(t)) \rho_{3} \eta_{3}(t)\right) \mathrm{d} t \\
& +b(O(t))\left(\rho_{1} \mathrm{~d} W_{1}(t)+\rho_{2} \mathrm{~d} W_{2}(t)+\rho_{3} W_{3}(t)\right),
\end{aligned}\right.
$$

where $\left(W_{\eta_{1}}(t), W_{\eta_{2}}(t), W_{\eta_{3}}(t), W_{\eta_{4}}(t)\right)$ are $Q^{\eta}$-Brownian motion defined as:

$$
\left\{\begin{array}{l}
\mathrm{d} W_{\eta_{1}}(t)=\mathrm{d} W_{1}(t)-\eta_{1}(t) \mathrm{d} t \\
\mathrm{~d} W_{\eta_{2}}(t)=\mathrm{d} W_{2}(t)-\eta_{2}(t) \mathrm{d} t, \\
\mathrm{~d} W_{\eta_{3}}(t)=\mathrm{d} W_{3}(t)-\eta_{3}(t) \mathrm{d} t, \\
\mathrm{~d} W_{\eta_{4}}(t)=\mathrm{d} W_{4}(t)-\eta_{4}(t) \mathrm{d} t .
\end{array}\right.
$$

Let $\mathcal{L}^{(\kappa, \eta)}$ be the differential operator given by 


$$
\begin{aligned}
\mathcal{L}^{(\kappa, \eta)} V(x, y, o, t) & =\frac{\partial V}{\partial t}+\left\{w_{2}\left(\left(\xi_{L}(o)-v\right)+\left(\sigma_{L}(o)-\beta \hat{\rho}_{2}\right) \eta_{2}\right)+w_{1} \xi_{S}(o)\right. \\
& \left.+\left(w_{1} \sigma_{1}(o)-\beta \hat{\rho}_{1} w_{2}\right) \eta_{1}-w_{2}\left(\beta \hat{\rho}_{3} \eta_{3}+\beta \hat{\rho}_{4} \eta_{4}\right)\right\} \frac{\partial V}{\partial x} \\
& +\left(\eta_{1}^{2}+\eta_{2}^{2}+\eta_{3}^{2}+\eta_{4}^{2}\right) y \frac{\partial V}{\partial y}+\left(a(o)+b(o) \rho_{1} \eta_{1}\right. \\
& \left.+b(o) \rho_{2} \eta_{2}+b(o) \rho_{3} \eta_{3}\right) \frac{\partial V}{\partial o}+\frac{1}{2}\left(\sigma_{1}^{2}(o) w_{1}^{2}-2 \beta \hat{\rho}_{1} \sigma_{1}(o) w_{1} w_{2}\right. \\
& \left.+\beta^{2} \hat{\rho}_{1}^{2} w_{2}^{2}+w_{2}^{2}\left(\sigma_{L}(o)-\beta \hat{\rho}_{2}\right)^{2}+w_{2}^{2} \beta^{2} \hat{\rho}_{3}^{2} w_{2}^{2} \beta^{2} \hat{\rho}_{4}^{2}\right) \frac{\partial^{2} V}{\partial x^{2}} \\
& +\frac{1}{2}\left(\eta_{1}^{2}+\eta_{2}^{2}+\eta_{3}^{2}+\eta_{4}^{2}\right) y^{2} \frac{\partial^{2} V}{\partial y^{2}}+\frac{1}{2} b^{2}(o) \frac{\partial^{2} V}{\partial o^{2}} \\
& +\left(\left(w_{1} \sigma_{1}(o)-\beta \hat{\rho}_{1} w_{2}\right) \eta_{1}+w_{2}\left(\sigma_{L}(o)-\beta \hat{\rho}_{2}\right) \eta_{2}\right. \\
& \left.-w_{2} \beta \hat{\rho}_{3} \eta_{3}-w_{2} \beta \hat{\rho}_{4} \eta_{4}\right) y \frac{\partial^{2} V}{\partial x \partial y}+\left(\left(w_{1} \sigma_{1}(o)-\beta \hat{\rho}_{1} w_{2}\right) b(o) \rho_{1}\right. \\
& \left.+w_{2}\left(\sigma_{L}(o)-\beta \hat{\rho}_{2}\right) b(o) \rho_{2}-w_{2} \beta \hat{\rho}_{3} b(o) \rho_{3}\right) \frac{\partial^{2} V}{\partial x \partial o} \\
& +\left(\eta_{1} b(o) \rho_{1}+\eta_{2} b(o) \rho_{2}+\eta_{3} b(o) \rho_{3}\right) y \frac{\partial^{2} V}{\partial y \partial o} .
\end{aligned}
$$

We now formulate a Verification Theorem. The proof of this theorem is similar to the proof of theorems from [18] [31] [32] [37]. For the completeness of this analysis we briefly state the theorem.

Theorem 1. (Verification Theorem). Suppose there exists a function $V \in \mathcal{C}^{2,2,2,1}(\mathbb{R} \times(0,+\infty) \times \mathbb{R} \times[0, T)) \cap \mathcal{C}(\mathbb{R} \times(0,+\infty) \times \mathbb{R} \times[0, T])$ and a Markov control $\left(\kappa^{*}(x, y, o, t), \eta^{*}(x, y, o, t)\right) \in \mathcal{A}_{t} \times \mathcal{M}$, such that

$$
\left\{\begin{array}{l}
\mathcal{L}^{\kappa^{*}(x, y, o, t), \eta} V(x, y, o, t) \leq 0, \\
\mathcal{L}^{\kappa, \eta^{*}(x, y, o, t)} V(x, y, o, t) \geq 0, \\
\mathcal{L}^{\kappa^{*}(x, y, o, t), \eta^{*}(x, y, o, t)} V(x, y, o, t)=0, \\
V(x, y, o, T)=-x-y
\end{array}\right.
$$

For all $\eta \in \mathbb{R}^{4}, \kappa \in \mathbb{R}^{2},(x, y, o, t) \in \mathbb{R} \times(0,+\infty) \times \mathbb{R} \times[0, T)$, and

$$
E_{x, y, o, t}^{\eta}\left[\sup _{t \leq s \leq T}\left|V\left(X^{\kappa}(s), Y(s), O(s), s\right)\right|\right]<+\infty
$$

For all $(x, y, o, t) \in \mathbb{R} \times[0,+\infty) \times \mathbb{R} \times[0, T], \kappa=\left(w_{1}, w_{2}\right) \in \mathcal{A}_{t}, \eta \in \mathcal{M}$. Then

$$
J^{\kappa^{*}, \eta}(x, y, o, t) \leq V(x, y, o, t) \leq J^{\kappa, \eta^{*}}(x, y, o, t)
$$

For all $\kappa=\left(w_{1}, w_{2}\right) \in \mathcal{A}_{t}, \eta \in \mathcal{M}$, and $V(x, y, o, t)=J^{\kappa^{*}, \eta^{*}}(x, y, o, t)$.

Proof. Fix $(x, y, o, t) \in \mathbb{R} \times(0,+\infty) \times \mathbb{R} \times[0, T)$, choose any $\eta \in \mathcal{M}$ and consider the system of Equation (21) with $\kappa^{*}(t)=\kappa^{*}(X(t), Y(t), O(t), t)$. If we apply the Itô formula to Equation (21) and the value function $V$, we obtain 


$$
\begin{aligned}
& E_{x, y, o, t}^{\eta}\left[V\left(X_{T_{n}^{\varepsilon} \wedge(T-\varepsilon)}^{\kappa^{*}}, Y_{T_{n}^{\varepsilon} \wedge(T-\varepsilon)}^{\eta}, O_{T_{n}^{\varepsilon} \wedge(T-\varepsilon)}, T_{n}^{\varepsilon} \wedge(T-\varepsilon)\right)\right] \\
& =V(x, y, o, t)+E_{x, y, o, t}^{\eta}\left[\int_{t}^{T_{n}^{\varepsilon} \wedge(T-\varepsilon)} \mathcal{L}^{\kappa^{*}(s), \eta(s)} V\left(X^{\kappa^{*}}(s), Y^{\eta}(s), O(s), s\right) \mathrm{d} s\right] \\
& \quad+E_{x, y, o, t}^{\eta}\left[\int_{t}^{T_{n}^{\varepsilon} \wedge(T-\varepsilon)} B^{\varepsilon}(s) \mathrm{d} W^{\eta}(s)\right]
\end{aligned}
$$

where $\left(T_{n}^{\varepsilon}, n=1,2, \cdots\right)$ is a localizing sequence of stopping times such that,

$$
E_{x, y, o, t}^{\eta}=\left[\int_{t}^{T_{n}^{\varepsilon} \wedge(T-\varepsilon)} B^{\varepsilon}(s) \mathrm{d} W^{\eta}(s)\right]=0 .
$$

Using $\mathcal{L}^{\kappa^{*}(x, y, o, t), \eta} V(x, y, o, t) \leq 0$, yields

$$
E_{x, y, o, t}^{\eta}\left[V\left(X_{T_{n}^{\varepsilon} \wedge(T-\varepsilon)}^{\kappa^{*}}, Y_{T_{n}^{\varepsilon} \wedge(T-\varepsilon)}^{\eta}, O_{T_{n}^{\varepsilon} \wedge(T-\varepsilon)}, T_{n}^{\varepsilon} \wedge(T-\varepsilon)\right)\right] \geq V(x, y, o, t) .
$$

Since Equation (25) holds, applying the dominated convergence theorem and letting $n \rightarrow+\infty$, and using $V(x, y, o, T)=-x-y$, we obtain $J^{\kappa^{*}, \eta}(x, y, o, t) \geq V(x, y, o, t)$. Replacing $\eta$ by $\eta^{*}$ and using $\mathcal{L}^{\kappa^{*}(x, y, o, t), \eta^{*}(x, y, o, t)} V(x, y, o, t)=0$, we obtain $J^{\kappa^{*}, \eta^{*}}(x, y, o, t)=V(x, y, o, t)$. Next choose any $\kappa \in \mathcal{A}_{t}$ and apply the Itô formula to the system of equations (21) with $\eta^{*}(t)=\eta^{*}(X(t), Y(t), O(t), t)$. Repeating the method presented above and using $\mathcal{L}^{\kappa, \eta^{*}(x, y, o, t)} V(x, y, o, t) \geq 0$, we obtain $J^{\kappa, \eta^{*}}(x, y, o, t) \leq V(x, y, o, t)$.

Remark 1. The conditions given in Equation (25) will hold if the upper and the lower HJBI equations are satisfied such that

$$
\begin{aligned}
& \min _{\kappa \in \mathbb{R}^{2}} \max _{\eta \in \mathbb{R}^{4}} \mathcal{L}^{(\kappa, \eta)} V(x, y, o, t)=\max _{\eta \in \mathbb{R}^{4}} \min _{\kappa \in \mathbb{R}^{2}} \mathcal{L}^{(\kappa, \eta)} V(x, y, o, t)=0, \\
& V(x, y, o, T)=-x-y .
\end{aligned}
$$

\subsection{Solution to the Stochastic Differential Equation}

To find the saddle point we first use the upper HJBI equation

$$
\min _{\kappa \in \mathbb{R}^{2}} \max _{\eta \in \mathbb{R}^{4}} \mathcal{L}^{(\kappa, \eta)} V(x, y, o, t)=0,
$$

Consequently, we obtain

$$
\begin{aligned}
& V_{t}+a(o) V_{o}+\frac{1}{2} b^{2}(o) V_{o o} \\
& +\min _{\kappa \in \mathbb{R}^{2}} \max _{\eta \in \mathbb{R}^{4}}\left\{\left[w_{2}\left(\left(\xi_{L}(o)-v\right)+\left(\sigma_{L}(o)-\beta \hat{\rho}_{2}\right) \eta_{2}-\beta \hat{\rho}_{3} \eta_{3}-\beta \hat{\rho}_{4}-\beta \hat{\rho}_{1} \eta_{1}\right)\right.\right. \\
& \left.+w_{1}\left(\xi_{S}(o)+\sigma_{1}(o) \eta_{1}\right)\right] V_{x}+\left(\eta_{1}^{2}+\eta_{2}^{2}+\eta_{3}^{2}+\eta_{4}^{2}\right) y V_{y} \\
& +b(o)\left(\rho_{1} \eta_{1}+\rho_{2} \eta_{2}+\rho_{3} \eta_{3}\right) V_{o}+\frac{1}{2}\left(\left(w_{1} \sigma_{1}(t)-\beta \hat{\rho}_{1} w_{2}\right)^{2}\right. \\
& \left.+w_{2}^{2}\left(\sigma_{L}(o)-\beta \hat{\rho}_{2}\right)^{2}+w_{2}^{2} \beta^{2} \hat{\rho}_{3}^{2}+w_{2}^{2} \beta^{2} \hat{\rho}_{4}^{2}\right) V_{x x}+\frac{1}{2}\left(\eta_{1}^{2}+\eta_{2}^{2}+\eta_{3}^{2}+\eta_{4}^{2}\right) y^{2} V_{y y} \\
& +\left(\left(w_{1} \sigma_{1}(o)-\beta \hat{\rho}_{1} w_{2}\right) \eta_{1}+w_{2}\left(\sigma_{L}(o)-\beta \hat{\rho}_{2}\right) \eta_{2}-w_{2} \beta \hat{\rho}_{3} \eta_{3}-w_{2} \beta \hat{\rho}_{4} \eta_{4}\right) y V_{x y} \\
& +b(o)\left(\left(w_{1} \sigma_{1}(o)-\beta \hat{\rho}_{1} w_{2}\right) \rho_{1}+w_{2}\left(\sigma_{L}(o)-\beta \hat{\rho}_{2}\right) \rho_{2}-w_{2} \beta \hat{\rho}_{3} \rho_{3}\right) V_{x o} \\
& \left.+b(o)\left(\eta_{1} \rho_{1}+\eta_{2} \rho_{2}+\eta_{3} \rho_{3}\right) y V_{y o}\right\}=0 .
\end{aligned}
$$


We expect $V(x, y, o, t)$ to be of the form

$$
V(x, y, o, t)=-x+G(o, t) y, \text { where } G(o, T)=-1 .
$$

Then we obtain

$$
\begin{aligned}
& y G_{t}+a(o) y G_{o}+\frac{1}{2} b^{2}(o) y G_{o o}+\min _{\kappa \in \mathbb{R}^{2}} \max _{\eta \in \mathbb{R}^{4}}\left\{\left[-w_{2}\left(\left(\xi_{L}(o)-v\right)\right.\right.\right. \\
& \left.\left.+\left(\sigma_{L}(o)-\beta \hat{\rho}_{2}\right) \eta_{2}-\beta \hat{\rho}_{1} \eta_{1}-\beta \hat{\rho}_{3} \eta_{3}-\beta \hat{\rho}_{4} \eta_{4}\right)-w_{1}\left(\xi_{S}(o)+\sigma_{1}(o) \eta_{1}\right)\right] \\
& \left.+2 b(o)\left(\rho_{1} \eta_{1}+\rho_{2} \eta_{2}+\rho_{3} \eta_{3}\right) y G_{o}\right\}=0 .
\end{aligned}
$$

The maximum for Equation (27) over $\left(\eta_{1}, \eta_{2}, \eta_{3}, \eta_{4}\right)$ is attained at $\left(\eta_{1}^{*}, \eta_{2}^{*}, \eta_{3}^{*}, \eta_{4}^{*}\right):$

$$
\begin{aligned}
& \eta_{1}^{*}\left(w_{1}, w_{2}\right)=\frac{\sigma_{1}(o)}{2 y G(o, t)} w_{1}-\frac{\beta \hat{\rho}_{1}}{2 y G(o, t)} w_{2}-b(o) \rho_{1} \frac{G_{o}(o, t)}{G(o, t)}, \\
& \eta_{2}^{*}\left(w_{2}\right)=\frac{\left(\sigma_{L}(o)-\beta \hat{\rho}_{2}\right)}{2 y G(o, t)} w_{2}-b(o) \rho_{2} \frac{G_{o}(o, t)}{G(o, t)}, \\
& \eta_{3}^{*}\left(w_{2}\right)=-\frac{\beta \hat{\rho}_{3}}{2 y G(o, t)} w_{2}-b(o) \rho_{3} \frac{G_{o}(o, t)}{G(o, t)}, \\
& \eta_{4}^{*}\left(w_{2}\right)=-\frac{\beta \hat{\rho}_{4}}{2 y G(o, t)} w_{2} .
\end{aligned}
$$

For $\left(\eta_{1}^{*}, \eta_{2}^{*}, \eta_{3}^{*}, \eta_{4}^{*}\right)$, we obtain

$$
\begin{aligned}
& y G_{t}+a(o) y G_{o}+\frac{1}{2} b^{2}(o) y G_{o o}+\min _{\kappa \in \mathbb{R}^{2}} \max _{\eta \in \mathbb{R}^{4}}\left\{\left[-w_{2}\left(\left(\xi_{L}(o)-v\right)\right.\right.\right. \\
& +\left(\sigma_{L}(o)-\beta \hat{\rho}_{2}\right) \eta_{2}^{*}\left(w_{2}\right)-\beta \hat{\rho}_{1} \eta_{1}^{*}\left(w_{1}, w_{2}\right)-\beta \hat{\rho}_{3} \eta_{3}^{*}\left(w_{2}\right) \\
& \left.\left.+\beta \hat{\rho}_{4} \eta_{4}^{*}\left(w_{2}\right)\right)-w_{1}\left(\xi_{S}(o)+\sigma_{1}(o) \eta_{1}^{*}\left(w_{1}, w_{2}\right)\right)\right] \\
& +\left(\left(\eta_{1}^{*}\left(w_{1}, w_{2}\right)\right)^{2}+\left(\eta_{2}^{*}\left(w_{2}\right)\right)^{2}+\left(\eta_{3}^{*}\left(w_{2}\right)\right)^{2}+\left(\eta_{4}^{*}\left(w_{2}\right)\right)^{2}\right) y G \\
& \left.+2 b(o)\left(\rho_{1} \eta_{1}^{*}\left(w_{1}, w_{2}\right)+\rho_{2} \eta_{2}^{*}\left(w_{2}\right)+\rho_{3} \eta_{3}^{*}\left(w_{2}\right)\right) y G_{o}\right\}=0 .
\end{aligned}
$$

Then the minimum over $\kappa:=\left(w_{1}, w_{2}\right)$ is attained at

$$
\begin{gathered}
w_{1}^{*}=-2 y G(o, t)\left[\frac{\xi_{S}(o)}{\sigma_{1}^{2}(o)}-2 \frac{\rho_{1} b(o)}{\sigma_{1}(o)} \frac{G_{o}(o, t)}{G(o, t)}\right]+\frac{\beta \hat{\rho}_{1}}{\sigma_{1}(o)} w_{2}^{*}, \\
w_{2}^{\star}=-2 y G(o, t)\left[\frac{\xi_{L}(o)-v}{\left(\sigma_{L}(o)-\beta \hat{\rho}_{2}\right)^{2}+\beta^{2}\left(\hat{\rho}_{1}^{2}+\hat{\rho}_{3}^{2}+\hat{\rho}_{4}^{2}\right)}\right. \\
\left.+\left(\frac{2 \beta b(o) \hat{\rho}_{1} \rho_{1}-b(o) \rho_{2}\left(\sigma_{L}(o)-\beta \hat{\rho}_{2}\right)+\beta b(o) \hat{\rho}_{3} \rho_{3}}{\left(\sigma_{L}(o)-\beta \hat{\rho}_{2}\right)^{2}+\beta^{2}\left(\hat{\rho}_{1}^{2}+\hat{\rho}_{3}^{2}+\hat{\rho}_{4}^{2}\right)}\right) \frac{G_{o}(o, t)}{G(o, t)}\right] \\
+\frac{\beta \hat{\rho}_{1} \sigma_{1}(o)}{\left(\sigma_{L}(o)-\beta \hat{\rho}_{2}\right)^{2}+\beta^{2}\left(\hat{\rho}_{1}^{2}+\hat{\rho}_{3}^{2}+\hat{\rho}_{4}^{2}\right)} w_{1}^{*} .
\end{gathered}
$$

By simplifying the above two equations, we obtain 


$$
\begin{aligned}
w_{1}^{*}= & -2 y G(o, t)\left[\frac{\Pi_{0} \xi_{S}(o)}{\sigma_{1}^{2}(o) \Pi_{2}}+\frac{\beta \hat{\rho}_{1}\left(\xi_{L}(o)-v\right)}{\sigma_{1}(o) \Pi_{2}}\right. \\
& \left.+\frac{\left(\beta \hat{\rho}_{1} \Pi_{1}-2 \rho_{1} b(o) \Pi_{0}\right)}{\sigma_{1}(o) \Pi_{2}} \frac{G_{o}(o, t)}{G(o, t)}\right], \\
w_{2}^{*}= & -2 y G(o, t)\left[\frac{\xi_{L}(o)-v}{\Pi_{2}}+\frac{\beta \hat{\rho}_{1} \xi_{S}(o)}{\sigma_{1}(o) \Pi_{2}}\right. \\
+ & \left.\left(\frac{\Pi_{1} \Pi_{2}+\beta^{2} \hat{\rho}_{1}^{2} \Pi_{1}-2 \beta b(o) \hat{\rho}_{1} \rho_{1} \Pi_{0}}{\Pi_{0} \Pi_{2}}\right) \frac{G_{o}(o, t)}{G(o, t)}\right],
\end{aligned}
$$

where

$$
\begin{gathered}
\Pi_{0}=\left(\sigma_{L}(o)-\beta \hat{\rho}_{2}\right)^{2}+\beta^{2}\left(\hat{\rho}_{1}^{2}+\hat{\rho}_{3}^{2}+\hat{\rho}_{4}^{2}\right), \\
\Pi_{2}=\left(\sigma_{L}(o)-\beta \hat{\rho}_{2}\right)^{2}+\beta^{2}\left(\hat{\rho}_{3}^{2}+\hat{\rho}_{4}^{2}\right)
\end{gathered}
$$

and

$$
\Pi_{1}=2 \beta b(o) \hat{\rho}_{1} \rho_{1}-b(o) \rho_{2}\left(\sigma_{L}(o)-\beta \hat{\rho}_{2}\right)+\beta b(o) \hat{\rho}_{3} \rho_{3} .
$$

Furthermore

$$
\begin{gathered}
\eta_{1}^{*}\left(w_{1}^{*}, w_{2}^{*}\right)=-\left(\frac{\xi_{S}(o)}{\sigma_{1}(o)}\right)-\left[\frac{\beta \hat{\rho}_{1} \Pi_{1}\left(\Pi_{1}-\Pi_{0}\right)-\rho_{1} b(o) \Pi_{0} \Pi_{2}}{\Pi_{0} \Pi_{2}}\right] \frac{G_{o}(o, t)}{G(o, t)}, \\
\eta_{2}^{*}\left(w_{2}^{*}\right)=-\left(\frac{\beta \hat{\rho}_{1}\left(\sigma_{L}(o)-\beta \hat{\rho}_{2}\right) \xi_{S}(o)}{\sigma_{1}(o) \Pi_{2}}\right)-\left(\frac{\left(\sigma_{L}(o)-\beta \hat{\rho}_{2}\right)\left(\xi_{L}(o)-v\right)}{\Pi_{2}}\right) \\
-\left(\frac{\left(\sigma_{L}(o)-\beta \hat{\rho}_{2}\right)\left(\Pi_{1}-2 \beta b(o) \hat{\rho}_{1} \rho_{1}\right)+b(o) \rho_{2} \Pi_{2}}{\Pi_{2}}\right) \frac{G_{o}(o, t)}{G(o, t)}, \\
\eta_{3}^{*}\left(w_{2}^{*}\right)=\left(\frac{\beta^{2} \hat{\rho}_{1} \hat{\rho}_{3} \xi_{S}(o)}{\left.\sigma_{1}(o) \Pi_{2}\right)+\left(\frac{\beta \hat{\rho}_{3}\left(\xi_{L}(o)-v\right)}{\Pi_{2}}\right)}\right. \\
+\left(\frac{\beta \hat{\rho}_{3} \Pi_{1}-b(o)\left(2 \beta^{2} \rho_{1} \hat{\rho}_{1} \hat{\rho}_{3}+\rho_{3} \Pi_{2}\right)}{\Pi_{2}}\right) \frac{G_{o}(o, t)}{G(o, t)}, \\
\eta_{4}^{*}\left(w_{2}^{*}\right)=\left(\frac{\beta^{2} \hat{\rho}_{1} \hat{\rho}_{4} \xi_{S}(o)}{\sigma_{1}(o) \Pi_{2}}\right)+\left(\frac{\beta \hat{\rho}_{4}\left(\xi_{L}(o)-v\right)}{\Pi_{2}}\right) \\
+\left(\frac{\beta \hat{\rho}_{4} \Pi_{1}-2 b(o) \beta^{2} \rho_{1} \hat{\rho}_{1} \hat{\rho}_{3}}{\Pi_{2}}\right) \frac{G_{o}(o, t)}{G(o, t)} .
\end{gathered}
$$

Then the saddle point candidate for the game $\left(\left(w_{1}^{*}, w_{2}^{*}\right), \eta_{1}^{*}\left(w_{1}, w_{2}\right), \eta_{2}^{*}\left(w_{2}\right), \eta_{3}^{*}\left(w_{2}\right), \eta_{4}^{*}\right)$, is governed by Equations (29), (30), (31), (32), (33) and (34). By substituting Equations (29) and (30) into Equation (28) and dividing by $y$, we obtain the final equation of the form

$$
G_{t}+\left(a(o)-d_{1}(o)\right) G_{o}+\frac{1}{2} b^{2}(o) G_{o o}-d_{2}(o) \frac{G_{o}^{2}}{G}+d_{3}(o) G=0,
$$

and we define $\lambda_{1}=\frac{\xi_{S}(o)}{\sigma_{1}(o)}, \lambda_{2}=\frac{\left(\xi_{L}(o)-v\right)}{\Pi_{2}}$. 


$$
\begin{aligned}
& d_{1}(o)=2 \frac{\left(\beta^{3} \hat{\rho}_{1} \hat{\rho}_{3}^{2}\right) \Pi_{1}}{\Pi_{2}^{2}} \lambda_{1}+8 \frac{\beta^{4} b(o) \rho_{1} \hat{\rho}_{1} \hat{\rho}_{3}}{\Pi_{2}^{2}} \lambda_{1}+4 \frac{\beta^{4} b(o) \rho_{1} \hat{\rho}_{1}^{2} \hat{\rho}_{3} \hat{\rho}_{4}}{\Pi_{2}^{2}} \lambda_{1} \\
& -4 b(o) \rho_{1} \lambda_{1}+4 \frac{\beta^{4} b(o) \rho_{1} \hat{\rho}_{1}^{2} \hat{\rho}_{4}^{2}}{\Pi_{2}^{2}} \lambda_{1}+2 \frac{\Pi_{0}}{\Pi_{2}} \rho_{1} b(o) \lambda_{1} \\
& -2 \frac{\beta^{3} \hat{\rho}_{1} \hat{\rho}_{4}^{2} \Pi_{1}}{\Pi_{0} \Pi_{2}} \lambda_{1}-2 \frac{\beta^{5} \hat{\rho}_{1}^{3} \hat{\rho}_{4}^{2} \Pi_{1}}{\Pi_{0} \Pi_{2}^{2}} \lambda_{1}+2 \frac{\Pi_{1} \Pi_{2}}{\Pi_{0}} \lambda_{2} \\
& +2 \frac{\beta^{2} \hat{\rho}_{1}^{2} \Pi_{1}}{\Pi_{0}} \lambda_{2}-2 \frac{\beta^{2} \hat{\rho}_{4}^{2} \Pi_{1}}{\Pi_{0}} \lambda_{2}-2 \frac{\beta^{4} \hat{\rho}_{1}^{2} \hat{\rho}_{4}^{2} \Pi_{1}}{\Pi_{0} \Pi_{2}} \lambda_{2} \\
& -4 \beta b(o) \rho_{1} \hat{\rho}_{1} \lambda_{2}+4 \frac{\beta^{3} b(o) \rho_{1} \hat{\rho}_{1} \hat{\rho}_{4}^{2}}{\Pi_{2}} \lambda_{2} \text {. } \\
& d_{2}(o)=\frac{\beta^{2} \hat{\rho}_{1}^{2} \Pi_{1}^{2}\left(\Pi_{1}-\Pi_{0}\right)^{2}}{\Pi_{0}^{2} \Pi_{2}^{2}}-4 \frac{\beta b(o) \rho_{1} \hat{\rho}_{1} \Pi_{1}\left(\Pi_{1}-\Pi_{0}\right)}{\Pi_{0} \Pi_{2}} \\
& -4 \beta^{3} b(o) \rho_{1} \hat{\rho}_{1} \hat{\rho}_{3}^{2} \frac{\Pi_{1}}{\Pi_{2}}+b^{2}(o)\left(3 \rho_{1}^{2}-\rho_{2}^{2}-\rho_{3}^{2}\right) \\
& +\frac{\Pi_{1}^{2}}{\Pi_{2}^{2}}\left(\beta^{2} \hat{\rho}_{3}^{2}+\beta^{2} \hat{\rho}_{4}^{2}\right) \frac{\Pi_{1}}{\Pi_{2}}+2\left(\frac{2 b(o) \beta^{2} \rho_{1} \hat{\rho}_{1} \hat{\rho}_{3}}{\Pi_{2}}\right)^{2} \\
& +\frac{\left(\sigma_{L}(o)-\beta \hat{\rho}_{2}\right)^{2}\left(\Pi_{1}-2 \beta b(o) \rho_{1} \hat{\rho}_{1}\right)}{\Pi_{2}}\left(\frac{\left(\Pi_{1}-2 \beta b(o) \rho_{1} \hat{\rho}_{1}\right)}{\Pi_{2}}\right. \\
& \left.-2 \Pi_{1}-2 \frac{\beta^{2} \hat{\rho}_{1} \Pi_{1}}{\Pi_{2}}+4 \frac{\beta b(o) \rho_{1} \hat{\rho}_{1}}{\Pi_{2}}\right) \\
& -2 \frac{b(o) \rho_{2}\left(\sigma_{L}(o)-\beta \hat{\rho}_{2}\right)}{\Pi_{0}}\left(\Pi_{1}+\frac{\beta^{2} \hat{\rho}_{1} \Pi_{1}}{\Pi_{2}}-\frac{2 \beta b(o) \rho_{1} \hat{\rho}_{1}}{\Pi_{2}}\right) \\
& -4 \frac{\beta^{3} b(o) \rho_{1} \hat{\rho}_{1} \hat{\rho}_{3} \hat{\rho}_{4} \Pi_{1}}{\Pi_{2}^{2}}-2 \frac{\beta^{2} \hat{\rho}_{3}^{2} \Pi_{1}}{\Pi_{0} \Pi_{2}}\left(\Pi_{1}+\frac{\beta^{2} \hat{\rho}_{1}^{2} \Pi_{1}}{\Pi_{2}}\right. \\
& \left.-2 \frac{\beta^{3} b(o) \rho_{1} \hat{\rho}_{1}^{3}}{\Pi_{2}}-\beta \hat{\rho}_{1}^{2} b(o)\right)+4 \frac{\beta^{2} b(o) \rho_{1} \hat{\rho}_{1} \hat{\rho}_{3}^{2}}{\Pi_{2}}\left(\beta \frac{\Pi_{1}}{\Pi_{2}}\right. \\
& \left.-2 \frac{\beta^{2} b(o) \rho_{1} \hat{\rho}_{1}}{\Pi_{2}}-b(o)\right)+2 \frac{\rho_{1} b(o)}{\Pi_{2}}\left(\beta \hat{\rho}_{1} \Pi_{1}-2 \rho_{1} b(o) \Pi_{0}\right) \\
& +4 \frac{b(o) \beta^{3} \rho_{1} \hat{\rho}_{1} \hat{\rho}_{3} \hat{\rho}_{4} \Pi_{1}}{\Pi_{2}}\left(\frac{1}{\Pi_{0}}+\frac{\beta^{2} \hat{\rho}_{1}^{2}}{\Pi_{0}}-2 \beta b(o) \rho_{1} \hat{\rho}_{1}\right) \\
& +2 b(o) \frac{\Pi_{1}}{\Pi_{0}}\left(\beta \hat{\rho}_{3}^{2}-\beta \rho_{1} \hat{\rho}_{1}-\frac{2 \beta^{3} \rho_{1} \hat{\rho}_{1} \hat{\rho}_{3}^{2}}{\Pi_{2}}\right) \\
& -2 \frac{\beta^{2} \hat{\rho}_{4}^{2} \Pi_{1}}{\Pi_{2}}\left(\frac{\Pi_{1}}{\Pi_{2}}-2 \frac{\beta b(o) \rho_{1} \hat{\rho}_{1}}{\Pi_{2}}+\frac{\beta^{2} \hat{\rho}_{1}^{2} \Pi_{1}}{\Pi_{0} \Pi_{2}}\right) \\
& +2 \beta^{2} \hat{\rho}_{1}^{2} \frac{\Pi_{1}\left(\Pi_{1}-\Pi_{0}\right)}{\Pi_{2}}\left(\frac{\beta^{3} \hat{\rho}_{1}^{3} \Pi_{1}}{\Pi_{0}^{2} \Pi_{2}}-2 \beta^{2} b(o) \rho_{1} \hat{\rho}_{1}^{2}+2 \frac{\rho_{1} b(o)}{\Pi_{2}}\right) \\
& -2 \beta b(o) \hat{\rho}_{1} \rho_{1}\left(\frac{\Pi_{1}}{\Pi_{0}}+\beta^{2} \hat{\rho}_{1}^{2} \frac{\Pi_{1}}{\Pi_{0} \Pi_{2}}-2 \beta \rho_{1} \hat{\rho}_{1} b(o) \Pi_{0}\right) .
\end{aligned}
$$




$$
\begin{aligned}
d_{3}(o)= & \lambda_{1}^{2}\left(1+2 \frac{\beta^{2} \hat{\rho}_{1}^{2}}{\Pi_{2}}-\frac{\beta^{2} \hat{\rho}_{1}^{2}\left(\sigma_{L}(o)-\beta \hat{\rho}_{2}\right)^{2}}{\Pi_{2}^{2}}-\frac{\left(\beta^{2} \hat{\rho}_{1} \hat{\rho}_{3}\right)^{2}}{\Pi_{2}^{2}}\right. \\
& \left.-\frac{\left(\beta^{2} \hat{\rho}_{1} \hat{\rho}_{4}\right)^{2}}{\Pi_{2}^{2}}\right)+\lambda_{2}^{2}\left(2 \Pi_{2}-\left(\sigma_{L}(o)-\beta \hat{\rho}_{2}\right)^{2}-\beta^{2} \hat{\rho}_{4}^{2}\right) \\
& +\lambda_{1} \lambda_{2}\left(4 \beta \hat{\rho}_{1}-2 \frac{\left(\sigma_{L}(o)-\beta \hat{\rho}_{2}\right)^{2} \beta \rho_{1}}{\Pi_{2}}-2 \frac{\beta^{3} \hat{\rho}_{1} \hat{\rho}_{3}^{2}}{\Pi_{2}}-2 \frac{\beta^{3} \hat{\rho}_{1} \hat{\rho}_{4}^{2}}{\Pi_{2}}\right) .
\end{aligned}
$$

Lemma 1. If the value function $V$ exists and is a solution to Equation (28), then it is also the solution to lower the Hamilton-Jacobi-Bellman-Isaacs (HJBI) equation $\max _{\eta \in \mathbb{R}^{4}} \min _{\kappa \in \mathbb{R}^{2}} \mathcal{L}^{(\kappa, \eta)} V(x, y, o, t)=0$.

Proof. We know that $\max _{\eta \in \mathbb{R}^{4}} \min _{\kappa \in \mathbb{R}^{2}} \mathcal{L}^{(\kappa, \eta)} V(x, y, o, t) \leq \min _{\kappa \in \mathbb{R}^{2}} \max _{\eta \in \mathbb{R}^{4}} \mathcal{L}^{(\kappa, \eta)} V(x, y, o, t)$, due to the fact that $\max _{\eta \in \mathbb{R}^{4}} \min _{\kappa \in \mathbb{R}^{2}} \mathcal{L}^{(\kappa, \eta)} V(x, y, o, t) \leq 0$. In addition we have

$$
\max _{\eta \in \mathbb{R}^{4}} \min _{\kappa \in \mathbb{R}^{2}} \mathcal{L}^{(\kappa, \eta)} V(x, y, o, t) \geq \min _{\kappa \in \mathbb{R}^{2}} \mathcal{L}^{(\kappa, \eta)} V(x, y, o, t), \forall \eta \in \mathbb{R}^{4} .
$$

Using Equations (35), (36), (37) and (38) we can verify that $\min _{\kappa \in \mathbb{R}^{2}} \mathcal{L}^{(\kappa, \eta)} V(x, y, o, t)=0$. This implies that $\max _{\eta \in \mathbb{R}^{4}} \min _{\kappa \in \mathbb{R}^{2}} \mathcal{L}^{(\kappa, \eta)} V(x, y, o, t) \geq 0$.

Lemma 2. Suppose that initial conditions $x_{0}, y_{0}, o_{0}, t_{0}$, are fixed and $G$ is a solution to Equation (35) and $\left(\left(w_{1}^{*}, w_{2}^{*}\right), \eta_{1}^{*}\left(w_{1}, w_{2}\right), \eta_{2}^{*}\left(w_{2}\right), \eta_{3}^{*}\left(w_{2}\right), \eta_{4}^{*}\right) \in \mathcal{A}_{t} \times \mathcal{M}$ is given by Equations (29), (30), (31), (32), (33) and (34). Then

$$
2 Y^{\eta^{*}}(t) G(O(t), t)=X^{\kappa^{*}}(t)-x_{0}+2 y_{0} G\left(o_{0}, t_{0}\right), \forall t \in[t(=0), T] .
$$

Proof. It is sufficient to prove that $\mathrm{d} X^{\kappa^{*}}(t)=\mathrm{d}\left(2 Y^{\eta^{*}}(t) G(O(t), t)\right)$. Due to the saddle point conditions the system of equation given by Equation (20), can be written as:

$$
\begin{aligned}
& \mathrm{d} X^{\left(\kappa^{*}\right)}(t) \\
& =-2 Y^{\eta^{*}}(t) G(O(t), t)\left[\frac{\Pi_{0} \lambda_{1}^{2}}{\Pi_{2}}+2 \beta \hat{\rho}_{1} \lambda_{1} \lambda_{2}+\frac{\left(\beta \hat{\rho}_{1} \Pi_{1}-2 \rho_{1} b(o) \Pi_{0}\right)}{\Pi_{2}} \lambda_{1} \frac{G_{o}(o, t)}{G(o, t)}\right. \\
& \left.+\Pi_{2} \lambda_{2}^{2}\left(\frac{\Pi_{1} \Pi_{2}+\beta^{2} \hat{\rho}_{1}^{2} \Pi_{1}-2 \beta b(o) \hat{\rho}_{1} \rho_{1} \Pi_{0}}{\Pi_{0}}\right) \lambda_{2} \frac{G_{o}(o, t)}{G(o, t)}\right] \mathrm{d} t \\
& -2 Y^{\eta^{*}}(t) G(O(t), t)\left[\left(\frac{\Pi_{0}}{\Pi_{2}}-\frac{\beta^{2} \hat{\rho}_{1}^{2}}{\Pi_{2}}\right) \lambda_{1}+\frac{\left(\beta \hat{\rho}_{1} \Pi_{1}-2 \rho_{1} b(o) \Pi_{0}\right)}{\Pi_{2}} \frac{G_{o}(o, t)}{G(o, t)}\right. \\
& \left.-\beta \hat{\rho}_{1}\left(\frac{\Pi_{1} \Pi_{2}+\beta^{2} \hat{\rho}_{1}^{2} \Pi_{1}-2 \beta b(o) \hat{\rho}_{1} \rho_{1} \Pi_{0}}{\Pi_{0} \Pi_{2}}\right) \frac{G_{o}(o, t)}{G(o, t)}\right] \mathrm{d} W_{1}(t) \\
& -2 Y^{\eta^{*}}(t) G(O(t), t)\left[\left(\sigma_{L}(o)-\beta \hat{\rho}_{2}\right) \lambda_{2}+\frac{\beta \hat{\rho}_{1}\left(\sigma_{L}(o)-\beta \hat{\rho}_{2}\right)}{\Pi_{2}} \lambda_{1}\right.
\end{aligned}
$$




$$
\begin{aligned}
& \left.+\left(\sigma_{L}(o)-\beta \hat{\rho}_{2}\right)\left(\frac{\Pi_{1} \Pi_{2}+\beta^{2} \hat{\rho}_{1}^{2} \Pi_{1}-2 \beta b(o) \hat{\rho}_{1} \rho_{1} \Pi_{0}}{\Pi_{0} \Pi_{2}}\right) \frac{G_{o}(o, t)}{G(o, t)}\right] \mathrm{d} W_{2}(t) \\
& +2 Y^{\eta^{*}}(t) G(O(t), t)\left[\beta \hat{\rho}_{3} \lambda_{2}+\frac{\beta^{2} \hat{\rho}_{1} \hat{\rho}_{3}}{\Pi_{2}} \lambda_{1}\right. \\
& \left.+\beta \hat{\rho}_{3}\left(\frac{\Pi_{1} \Pi_{2}+\beta^{2} \hat{\rho}_{1}^{2} \Pi_{1}-2 \beta b(o) \hat{\rho}_{1} \rho_{1} \Pi_{0}}{\Pi_{0} \Pi_{2}}\right) \frac{G_{o}(o, t)}{G(o, t)}\right] \mathrm{d} W_{3}(t) \\
& +2 Y^{\eta^{*}}(t) G(O(t), t)\left[\beta \hat{\rho}_{4} \lambda_{2}+\frac{\beta^{2} \hat{\rho}_{1} \hat{\rho}_{4}}{\Pi_{2}} \lambda_{1}\right. \\
& \left.+\beta \hat{\rho}_{4}\left(\frac{\Pi_{1} \Pi_{2}+\beta^{2} \hat{\rho}_{1}^{2} \Pi_{1}-2 \beta b(o) \hat{\rho}_{1} \rho_{1} \Pi_{0}}{\Pi_{0} \Pi_{2}}\right) \frac{G_{o}(o, t)}{G(o, t)}\right] \mathrm{d} W_{4}(t),
\end{aligned}
$$

and

$$
\begin{aligned}
& \mathrm{d} Y^{\eta^{*}} \\
& =-\left[\lambda_{1} Y^{\eta^{*}}+\left(\frac{\beta \hat{\rho}_{1} \Pi_{1}\left(\Pi_{1}-\Pi_{0}\right)-\rho_{1} b(o) \Pi_{0} \Pi_{2}}{\Pi_{0} \Pi_{2}}\right) Y^{\eta^{*}} \frac{G_{o}(o, t)}{G(o, t)}\right] \mathrm{d} W_{1}(t) \\
& -\left[\left(\frac{\beta \hat{\rho}_{1}\left(\sigma_{L}(o)-\beta \hat{\rho}_{2}\right)}{\Pi_{2}}\right) \lambda_{1} Y^{\eta^{*}}+\left(\sigma_{L}(o)-\beta \hat{\rho}_{2}\right) \lambda_{2} Y^{\eta^{*}}\right. \\
& \left.+\left(\frac{\left(\sigma_{L}(o)-\beta \hat{\rho}_{2}\right)\left(\Pi_{1}-2 \beta b(o) \hat{\rho}_{1} \rho_{1}\right)+b(o) \rho_{2} \Pi_{2}}{\Pi_{2}}\right) Y^{\eta^{*}} \frac{G_{o}(o, t)}{G(o, t)}\right] \mathrm{d} W_{2}(t) \\
& +\left[\frac{\beta^{2} \hat{\rho}_{1} \hat{\rho}_{3}}{\Pi_{2}} \lambda_{1} Y^{\eta^{\star}}+\beta \hat{\rho}_{3} \lambda_{2} Y^{\eta^{*}}\right. \\
& \left.+\left(\frac{\beta \hat{\rho}_{3} \Pi_{1}-b(o)\left(2 \beta^{2} \rho_{1} \hat{\rho}_{1} \hat{\rho}_{3}+\rho_{3} \Pi_{2}\right)}{\Pi_{2}}\right) Y^{\eta^{*}} \frac{G_{o}(o, t)}{G(o, t)}\right] \mathrm{d} W_{3}(t) \\
& +\left[\frac{\beta^{2} \hat{\rho}_{1} \hat{\rho}_{4}}{\Pi_{2}} \lambda_{1} Y^{\eta^{*}}+\beta \hat{\rho}_{4} \lambda_{2} Y^{\eta^{*}}\right. \\
& \left.+\left(\frac{\beta \hat{\rho}_{4} \Pi_{1}-2 b(o) \beta^{2} \rho_{1} \hat{\rho}_{1} \hat{\rho}_{3}}{\Pi_{2}}\right) Y^{\eta^{*}} \frac{G_{o}(o, t)}{G(o, t)}\right] \mathrm{d} W_{4}(t) .
\end{aligned}
$$

Using Equation (35) we can verify that

$$
\begin{aligned}
& \mathrm{d} G(O(t), t) \\
& =\left[d_{1}(O(t), t)+d_{2}(O(t), t)-d_{3}(O(t), t)\right] \mathrm{d} t \\
& \quad+G_{0}(O(t), t) b(O(t))\left(\hat{\rho}_{1} \mathrm{~d} W_{1}(t)+\hat{\rho}_{2} \mathrm{~d} W_{2}(t)+\hat{\rho}_{3} W_{3}(t)+\hat{\rho}_{4} W_{4}(t)\right),
\end{aligned}
$$

where $d_{1}(O(t), t), d_{2}(O(t), t)$ and $d_{3}(o(t), t)$ are defined above Equations (36), (37) and (38). We obtain the right hand side of Equation (40), due to the fact that

$$
\begin{aligned}
\mathrm{d}\left(2 Y^{\eta^{*}} G(O(t), t)\right)= & 2 G(O(t), t) \mathrm{d} Y^{\eta^{*}}(t)+2 Y^{\eta^{*}}(t) \mathrm{d} G(O(t), t) \\
& +2 \mathrm{~d} Y^{\eta^{*}}(t) \mathrm{d} G(O(t), t) .
\end{aligned}
$$




\subsection{Economic Analysis}

In this subsection, we analyze the impact of $\hat{\rho}_{1}$ and $\hat{\rho}_{2}$ on the optimal policies. For fixed initial conditions $\left(x_{0}, y_{0}, o_{0}, t_{0}\right)$ under Lemma 2 ensures that

$$
\begin{aligned}
w_{1}^{*}= & -2 Y^{\eta^{*}}(t) G(O(t), t)\left[\frac{\Pi_{0} \xi_{S}(o)}{\sigma_{1}^{2}(o) \Pi_{2}}+\frac{\beta \hat{\rho}_{1}\left(\xi_{L}(o)-v\right)}{\sigma_{1}(o) \Pi_{2}}\right. \\
& \left.+\frac{\left(\beta \hat{\rho}_{1} \Pi_{1}-2 \rho_{1} b(o) \Pi_{0}\right)}{\sigma_{1}(o) \Pi_{2}} \frac{G_{o}(o, t)}{G(o, t)}\right], \\
w_{2}^{*}= & -2 Y^{\eta^{*}}(t) G(o(t), t)\left[\frac{\xi_{L}(o)-v}{\Pi_{2}}+\frac{\beta \hat{\rho}_{1} \xi_{S}(o)}{\sigma_{1}(o) \Pi_{2}}\right. \\
& \left.+\left(\frac{\Pi_{1} \Pi_{2}+\beta^{2} \hat{\rho}_{1}^{2} \Pi_{1}-2 \beta b(o) \hat{\rho}_{1} \rho_{1} \Pi_{0}}{\Pi_{0} \Pi_{2}}\right) \frac{G_{o}(o, t)}{G(o, t)}\right] .
\end{aligned}
$$

Hence we can use

$$
\begin{aligned}
\hat{\kappa}^{*}= & -\left(X^{\kappa^{*}}(t)-x_{0}+2 y_{0} G\left(o_{0}, t_{0}\right)[\underbrace{\frac{\Pi_{0} \lambda_{1}}{\sigma_{1}(o) \Pi_{2}}+\frac{\beta \hat{\rho}_{1} \lambda_{2}}{\sigma_{1}(o)}}_{\text {myopicdemand }}\right. \\
& +\underbrace{\left.\frac{\left(\beta \hat{\rho}_{1} \Pi_{1}-2 \rho_{1} b(o) \Pi_{0}\right)}{\sigma_{1}(o) \Pi_{2}} \frac{G_{o}(o, t)}{G(o, t)}\right]+2 y_{0} G\left(o_{0}, t_{0}\right)}_{\text {intertemporal hedging demand }}] \underbrace{\lambda_{2}+\frac{\beta \hat{\rho}_{1} \lambda_{1}}{\Pi_{2}}}_{\text {myopicdemand }} \\
& +\underbrace{\left.\frac{\Pi_{1} \Pi_{2}+\beta^{2} \hat{\rho}_{1}^{2} \Pi_{1}-2 \beta b(o) \hat{\rho}_{1} \rho_{1} \Pi_{0}}{\Pi_{0} \Pi_{2}}\right) \frac{G_{o}(o, t)}{G(o, t)}}_{\text {intertemporal hedging demand }}])
\end{aligned}
$$

1) Each optimal portfolio weight is a sum of two terms, the first being the myopically optimal portfolio. It is a sum of a two factors and depends on the ratio of the first to second moments of excess returns and the impacts of $\hat{\rho}_{1}, \beta$, $\Pi_{0}$ and $\Pi_{2}$. It corresponds to the instantaneous mean-variance portfolio in which the investment opportunity set remain constant through time. The myopic portfolio is always positive for a nonzero market price of risk. (Myopic mean variance demand does not include intertemporal hedging component). This myopic term is well-known from Merton's problem and can be reproduced in our general setup of stochastic coefficients [38].

2) The second term, intertemporal hedging portfolio is the portfolio with the maximal absolute correlation with the state variable (inflation). It represents the difference between the solution under stochastic coefficients and the myopic solution and the additional investment is caused by the presence of the stochastic factor (inflation). It does not have a constant sign. The excess risky demand vanishes in the uncorrelated case $\hat{\rho}_{1}=\rho_{1}=\rho_{2}=\rho_{3}=\hat{\rho}_{3}=0$ and when the volatility of the inflation process is zero. For $w_{1}^{*}$ it is a sum of two factors whilst for $w_{2}^{*}$ 
it is a sum of three factors and $\frac{G_{o}(o, t)}{G(o, t)}$ measures the importance of the state variable is to the banker. The myopic part, these portfolios are weighted by the inverse of the banker's risk aversion. Hence, the optimization of our modified version of monotone mean-variance preferences is consistent with classical mean-variance optimization subject to a suitably chosen risk aversion parameter $\gamma$, (which depend on $o_{0}$ ). When the risk aversion $\gamma\left(o_{0}\right)>1$, we have a positive intertemporal hedging demand for risky assets and it exhibits a hump-shaped function of risk tolerance $\frac{1}{\gamma\left(o_{0}\right)}$. This is due to the fact that investors with unit risk aversion have no intertemporal hedging demand. It is important to note that both the myopic and hedging demands are scaled equally by risk aversion and that the trade-off between holding a myopically optimal portfolio and intertemporal hedging is determined by the derivatives of marginal utility with respect to the state variables. Intertemporal hedging portfolio strategy may hedge or speculate on expected return or mean-aversion risk by choosing to hold long or short position of the risky assets.

3) $\hat{\rho}_{1}>0$, examines the impact of banker's provision capital risk process on the optimal stock index return holding portfolio $w_{1}^{*}$. Since the myopic portfolio demand for risky assets are always a linear function of risk tolerance $\frac{1}{\gamma\left(o_{0}\right)}$. When $\hat{\rho}_{1}>0$ and $\Pi_{0} \lambda_{1}+\sigma_{1}(o) \beta \hat{\rho}_{1} \lambda_{2} \Pi_{2}>\left(\beta \hat{\rho}_{1} \Pi_{1}-2 \rho_{1} b(o) \Pi_{0}\right)$, the intertemporal hedging demand on the optimal stock index fund is smaller than the myopic portfolio. Intuitively, the banker takes long positions if $\frac{\left(\beta \hat{\rho}_{1} \Pi_{1}-2 \rho_{1} b(o) \Pi_{0}\right)}{\sigma_{1}(o) \Pi_{2}}<0$, or short positions if $\frac{\left(\beta \hat{\rho}_{1} \Pi_{1}-2 \rho_{1} b(o) \Pi_{0}\right)}{\sigma_{1}(o) \Pi_{2}}>0$ in each of the perfectly correlated portfolios to hedge against undesirable innovations of the market state variable in the intertemporal hedging demand portfolio.

4) On the other hand if $\hat{\rho}_{1}<0$, implies a negative impact on optimal stock index return holding portfolio $w_{1}^{*}$ due to banker's provision capital risk process. If $\hat{\rho}_{1}<0$ and $\Pi_{0}\left(\Pi_{2} \lambda_{2}+\beta \hat{\rho}_{1} \lambda_{1}\right)>\left(\Pi_{1} \Pi_{2}+\beta^{2} \hat{\rho}_{1} \Pi_{1}-2 \beta b(o) \hat{\rho}_{1} \rho_{1} \Pi_{0}\right)$ the intertemporal hedging demand on the optimal stock index fund is smaller than the myopic portfolio. Intuitively the banker takes long positions if $\left(\Pi_{1} \Pi_{2}+\beta^{2} \hat{\rho}_{1} \Pi_{1}-2 \beta b(o) \hat{\rho}_{1} \rho_{1} \Pi_{0}\right)<0$ or short positions $\left(\Pi_{1} \Pi_{2}+\beta^{2} \hat{\rho}_{1} \Pi_{1}-2 \beta b(o) \hat{\rho}_{1} \rho_{1} \Pi_{0}\right)>0$ in each of the perfectly correlated portfolios to hedge against undesirable innovations of the market state variable in the intertemporal hedging demand portfolio.

5) To examine the impact of $\hat{\rho}_{1}>0$, on optimal loan return portfolio $w_{2}^{*}$, we set $\hat{\rho}_{1}>0$ and $\lambda_{2}+\frac{\beta \hat{\rho}_{1} \lambda_{1}}{\Pi_{2}}>\frac{\Pi_{1} \Pi_{2}+\beta^{2} \hat{\rho}_{1}^{2} \Pi_{1}-2 \beta b(o) \hat{\rho}_{1} \rho_{1} \Pi_{0}}{\Pi_{0} \Pi_{2}}$. Then the intertemporal hedging demand of the optimal loan portfolio is smaller than the myopic portfolio. Intuitively, the banker takes long positions if

$\frac{\Pi_{1} \Pi_{2}+\beta^{2} \hat{\rho}_{1}^{2} \Pi_{1}-2 \beta b(o) \hat{\rho}_{1} \rho_{1} \Pi_{0}}{\Pi_{0} \Pi_{2}}<0$, or short positions if 
$\frac{\Pi_{1} \Pi_{2}+\beta^{2} \hat{\rho}_{1}^{2} \Pi_{1}-2 \beta b(o) \hat{\rho}_{1} \rho_{1} \Pi_{0}}{\Pi_{0} \Pi_{2}}>0$, in each of the perfectly correlated portfolios to hedge against undesirable innovations of the market state variable in the intertemporal hedging demand portfolio.

6) If $\hat{\rho}_{1}<0$, and $\lambda_{2}+\frac{\beta \hat{\rho}_{1} \lambda_{1}}{\Pi_{2}}>\frac{\Pi_{1} \Pi_{2}+\beta^{2} \hat{\rho}_{1}^{2} \Pi_{1}-2 \beta b(o) \hat{\rho}_{1} \rho_{1} \Pi_{0}}{\Pi_{0} \Pi_{2}}$, the intertemporal hedging demand on the optimal loan portfolio is smaller than the myopic portfolio. Intuitively, the banker takes short positions if

$\frac{\Pi_{1} \Pi_{2}+\beta^{2} \hat{\rho}_{1}^{2} \Pi_{1}-2 \beta b(o) \hat{\rho}_{1} \rho_{1} \Pi_{0}}{\Pi_{0} \Pi_{2}}>0$ or long position

$\frac{\Pi_{1} \Pi_{2}+\beta^{2} \hat{\rho}_{1}^{2} \Pi_{1}-2 \beta b(o) \hat{\rho}_{1} \rho_{1} \Pi_{0}}{\Pi_{0} \Pi_{2}}<0$ in each of the perfectly correlated portfolios to hedge against undesirable innovations of the market state variable in the intertemporal hedging demand portfolio.

7) If $\hat{\rho}_{2}>0$, will also affect the magnitudes of optimal portfolios via $\Pi_{0}, \Pi_{1}$ and $\Pi_{2}$. If $\hat{\rho}_{2}>0$ and $\Pi_{0} \lambda_{1}+\beta \hat{\rho}_{1} \lambda_{2} \Pi_{2}>\left(\beta \hat{\rho}_{1} \Pi_{1}-2 \rho_{1} b(o) \Pi_{0}\right)$, then the intertemporal hedging demand on the optimal stock index fund, $w_{1}^{*}$ is smaller than the myopic portfolio. Intuitively, the banker takes long positions if $\frac{\left(\beta \hat{\rho}_{1} \Pi_{1}-2 \rho_{1} b(o) \Pi_{0}\right)}{\sigma_{1}(o) \Pi_{2}}<0$, or short positions if $\frac{\left(\beta \hat{\rho}_{1} \Pi_{1}-2 \rho_{1} b(o) \Pi_{0}\right)}{\sigma_{1}(o) \Pi_{2}}>0$, in each of the perfectly correlated portfolios to hedge against undesirable innovations of the market state variable in the intertemporal hedging demand portfolio.

$8)$ If $\hat{\rho}_{2}<0$ and $\Pi_{0}\left(\Pi_{2} \lambda_{2}+\beta \hat{\rho}_{1} \lambda_{1}\right)>\left(\Pi_{1} \Pi_{2}+\beta^{2} \hat{\rho}_{1}^{2} \Pi_{1}-2 \beta b(o) \hat{\rho}_{1} \rho_{1} \Pi_{0}\right)$ the intertemporal hedging demand for the optimal stock index fund $w_{1}^{*}$ is smaller than the myopic portfolio. Intuitively, the banker takes long positions if $\left(\Pi_{1} \Pi_{2}+\beta^{2} \hat{\rho}_{1}^{2} \Pi_{1}-2 \beta b(o) \hat{\rho}_{1} \rho_{1} \Pi_{0}\right)<0$, or short positions $\left(\Pi_{1} \Pi_{2}+\beta^{2} \hat{\rho}_{1}^{2} \Pi_{1}-2 \beta b(o) \hat{\rho}_{1} \rho_{1} \Pi_{0}\right)>0$, in each of the perfectly correlated portfolios to hedge against undesirable innovations in the market state variable in the intertemporal hedging demand portfolio.

9) If $\hat{\rho}_{2}>0$ and $\lambda_{2}+\frac{\beta \hat{\rho}_{1} \lambda_{1}}{\Pi_{2}}>\frac{\Pi_{1} \Pi_{2}+\beta^{2} \hat{\rho}_{1}^{2} \Pi_{1}-2 \beta b(o) \hat{\rho}_{1} \rho_{1} \Pi_{0}}{\Pi_{0} \Pi_{2}}$, the intertemporal hedging demand on the optimal loan portfolio $w_{2}^{*}$ is smaller than the myopic portfolio. Intuitively, the banker takes long positions if $\frac{\Pi_{1} \Pi_{2}+\beta^{2} \hat{\rho}_{1}^{2} \Pi_{1}-2 \beta b(o) \hat{\rho}_{1} \rho_{1} \Pi_{0}}{\Pi_{0} \Pi_{2}}<0$, or short positions if

$\frac{\Pi_{1} \Pi_{2}+\beta^{2} \hat{\rho}_{1}^{2} \Pi_{1}-2 \beta b(o) \hat{\rho}_{1} \rho_{1} \Pi_{0}}{\Pi_{0} \Pi_{2}}>0$ in each of the perfectly correlated portfolios to hedge against undesirable innovations of the market state variable in the intertemporal hedging demand portfolio.

10) If $\hat{\rho}_{2}<0$ and $\lambda_{2}+\frac{\beta \hat{\rho}_{1} \lambda_{1}}{\Pi_{2}}>\frac{\Pi_{1} \Pi_{2}+\beta^{2} \hat{\rho}_{1}^{2} \Pi_{1}-2 \beta b(o) \hat{\rho}_{1} \rho_{1} \Pi_{0}}{\Pi_{0} \Pi_{2}}$, the intertemporal hedging demand of the optimal loan portfolio is smaller than the myopic portfolio. Intuitively, the banker takes short positions 
$\frac{\Pi_{1} \Pi_{2}+\beta^{2} \hat{\rho}_{1}^{2} \Pi_{1}-2 \beta b(o) \hat{\rho}_{1} \rho_{1} \Pi_{0}}{\Pi_{0} \Pi_{2}}>0$, or long positions if

$\frac{\Pi_{1} \Pi_{2}+\beta^{2} \hat{\rho}_{1}^{2} \Pi_{1}-2 \beta b(o) \hat{\rho}_{1} \rho_{1} \Pi_{0}}{\Pi_{0} \Pi_{2}}<0$, in each of the perfectly correlated port-

folios to hedge against undesirable innovations of the market state variables in the intertemporal hedging demand portfolio.

Note:

$\hat{\rho}_{1}>0$ implies $\Pi_{0}>0, \Pi_{2}>0$ and $\Pi_{1}>0$, iff

$$
\left(2 \beta b(o) \hat{\rho}_{1} \rho_{1}-b(o) \rho_{2}\left(\sigma_{L}(o)-\beta \hat{\rho}_{2}\right)\right)<\beta b(o) \hat{\rho}_{3} \rho_{3} .
$$

$\hat{\rho}_{1}<0$ implies $\Pi_{0}>0, \Pi_{2}>0$ and $\Pi_{1}>0$ iff

$$
\left(2 \beta b(o) \hat{\rho}_{1} \rho_{1}-b(o) \rho_{2}\left(\sigma_{L}(o)-\beta \hat{\rho}_{2}\right)\right)<\beta b(o) \hat{\rho}_{3} \rho_{3} .
$$

$\hat{\rho}_{2}>0$ implies $\Pi_{0}>0, \Pi_{2}>0$ and $\Pi_{1}>0$, iff $\left(\sigma_{L}(o)-\beta \hat{\rho}_{2}\right)<0$.

$\hat{\rho}_{2}<0$ implies $\Pi_{0}>0, \Pi_{2}>0$ and $\Pi_{1}>0$.

\subsection{Smooth Solution to the Resulting Equation}

In order to obtain a smooth solution to Equation (35) we follow [39] and obtain the following results subject to the boundary condition $G(o, T)=-1$.

Case 1: $d_{2} \neq \frac{b^{2}(o)}{2}$. Define $G(o, t)=-F^{\alpha}(o, t)$, where $F(z, T)=1$, to obtain

$$
\begin{aligned}
& F_{t}+\left(a(o)-d_{1}(o)\right) F_{o}+\frac{1}{2} b^{2}(o) F_{o o} \\
& +\left[\frac{1}{2} b^{2}(o)(\alpha-1)-\alpha d_{2}(o)\right] \frac{F_{o}^{2}}{F}+\frac{1}{\alpha} d_{3}(o) F=0 .
\end{aligned}
$$

This implies that $\alpha=\left(\frac{b^{2}(o)}{b^{2}(o)-2 d_{2}(o)}\right)$ and we obtain

$$
F_{t}+\left(a(o)-d_{1}(o)\right) F_{o}+\frac{1}{2} b^{2}(o) F_{o o}+\left(\frac{b^{2}(o)-2 d_{2}(o)}{b^{2}(o)}\right) d_{3}(o) F=0 .
$$

Case I1: $d_{2}=\frac{b^{2}(o)}{2}$. Define $G(o, t)=e^{F(o, t)}$, where $F(z, T)=0$, to obtain

$$
F_{t}+\left(a(o)-d_{1}(o)\right) F_{o}+\frac{1}{2} b^{2}(o) F_{o o}+d_{3}(o) F=0 .
$$

Remark 2. If $a, b, \sum_{i=1}^{2} b \cdot \lambda_{i}, \sum_{i=1}^{2} \lambda_{i}^{2}$ are Lipschitz continuous, $\sum_{i=1}^{2} \lambda_{i}$ are continuous and bounded by $0<\varepsilon<b^{2}$, then there exists unique smooth solutions for $F_{1}$ and $F_{2}$ to Equations (42) and (43) respectively via Theorem 1 of [40]. $F_{1}$ and $F_{2}$ satisfy the Feynman-Kac representations such that

$$
\begin{aligned}
& F_{1}(o, t)=\mathbb{E}_{o, t}\left[\exp \left\{\left(\frac{b^{2}(o)-2 d_{2}(o)}{b^{2}(o)}\right) \int_{t}^{T} d_{3}(\tilde{O}(s)) \mathrm{d} s\right\}\right], \\
& F_{2}(o, t)=\mathbb{E}_{o, t}\left[\int_{t}^{T} d_{3}(\tilde{O}(s)) \mathrm{d} s\right],
\end{aligned}
$$


where $\mathrm{d} \tilde{O}(s)=\left[a(\tilde{O}(s))-d_{1}(\tilde{O}(s))\right] \mathrm{d} s+b(\tilde{O}(s)) \mathrm{d} W(s) \quad$ and $\quad \tilde{O}(t)=o$ Since, $\sum_{i=1}^{2} \lambda_{i}$ are bounded functions it implies that $F_{1}$ and $F_{2}$ are bounded and $G$ is bounded away from zero for any $\left(\rho_{1}, \rho_{2}, \rho_{3}\right) \in[-1,1]$.

Lemma 3. Suppose $a, b, \sum_{i=1}^{2} b \cdot \lambda_{i}, \sum_{i=1}^{2} \lambda_{i}^{2}$ are Lipschitz continuous, $\sum_{i=1}^{2} \lambda_{i}$ are continuous and bounded as $0<\varepsilon<b^{2}$ and $F$ is a bounded solution to Equation (42) or Equation (43). Then the first order o-derivative of $F$ is bounded.

Proof. To obtain a bound for $F_{o}$, it is sufficient to estimate the Lipschitz constant. First of all, noting that $\left(o_{1}, o_{2}\right) \in(-\infty, a]$, there exists $L_{a}>0$ such that $\left|e^{o_{1}}-e^{o_{2}}\right| \leq L_{a}|o-\bar{o}|$.

Secondly, the solution to Equations (42) and (43) and the fact that $\lambda_{1}^{2}$ and $\lambda_{2}^{2}$ are Lipschitz continuous and bounded, we obtain the existence of $L>0$, such that

$$
\begin{aligned}
|F(o, t)-F(\bar{o}, t)| & \leq L \mathbb{E}\left[\int_{t}^{T}\left|\tilde{F}_{s}(o, t)-\tilde{F}_{s}(\bar{o}, t)\right| \mathrm{d} s\right] \\
& \leq L T \mathbb{E}\left[\sup _{t \leq s \leq T}\left|\tilde{F}_{s}(o, t)-\tilde{F}_{s}(\bar{o}, t)\right|\right],
\end{aligned}
$$

where from notation convenience, we write $\mathbb{E} f\left(\tilde{F}_{s}(o, t)\right)$ instead of $\mathbb{E}_{o, t} f\left(\tilde{F}_{s}\right)$. Then via (Theorem 1.3.16 of Pham [41], there exists $C_{T}>0$, such that $\mathbb{E}\left[\sup _{t \leq s \leq T}\left|\tilde{F}_{s}(o, t)-\tilde{F}_{s}(\bar{o}, t)\right|\right] \leq C_{T}|o-\bar{o}|$, which completes the proof in the first case. Similar estimate can be deriving for the solution for Equation (43).

Theorem 2. Suppose $a, b, \sum_{i=1}^{2} b \cdot \lambda_{i}, \sum_{i=1}^{2} \lambda_{i}^{2}$, are Lipschitz continuous, $\sum_{i=1}^{2} \lambda_{i}$ are continuous and bounded, $0<\epsilon<b^{2}$. Then there exists a saddle point $\kappa^{*}(x, y, o, t), \eta^{*}(x, y, o, t) \in \mathcal{A}_{t} \times \mathcal{M}$, for problem (19) such that

$$
\begin{aligned}
w_{1}^{*}=-2 y G(o, t) & {\left[\frac{\Pi_{0} \lambda_{1}(o)}{\sigma_{1}(0) \Pi_{2}}+\frac{\beta \hat{\rho}_{1} \lambda_{2}(o)}{\sigma_{1}(o)}+\frac{\left(\beta \hat{\rho}_{1} \Pi_{1}-2 \rho_{1} b(o) \Pi_{0}\right)}{\sigma_{1}(o) \Pi_{2}} \frac{G_{o}(o, t)}{G(o, t)}\right], } \\
w_{2}^{*}= & -2 y G(o, t)\left[\lambda_{2}(o)+\frac{\beta \hat{\rho}_{1} \lambda_{1}(o)}{\Pi_{2}}\right. \\
& \left.+\left(\frac{\Pi_{1} \Pi_{2}+\beta^{2} \hat{\rho}_{1}^{2} \Pi_{1}-2 \beta b(o) \hat{\rho}_{1} \rho_{1} \Pi_{0}}{\Pi_{0} \Pi_{2}}\right) \frac{G_{o}(o, t)}{G(o, t)}\right], \\
\eta_{1}^{*}\left(w_{1}^{*}, w_{2}^{*}\right)= & -\left(\frac{1}{\sigma_{1}(o)}\right) \xi_{S}(o)-\left[\frac{\beta \hat{\rho}_{1} \Pi_{1}\left(\Pi_{1}-\Pi_{0}\right)-\rho_{1} b(o) \Pi_{0} \Pi_{2}}{\Pi_{0} \Pi_{2}}\right] \frac{G_{o}(o, t)}{G(o, t)}, \\
\eta_{2}^{*}\left(w_{2}^{*}\right)= & -\left(\frac{\beta \hat{\rho}_{1}\left(\sigma_{L}(o)-\beta \hat{\rho}_{2}\right) \xi_{S}(o)}{\sigma_{1}(o) \Pi_{2}}\right)-\left(\frac{\left(\sigma_{L}(o)-\beta \hat{\rho}_{2}\right)\left(\xi_{L}(o)-v\right)}{\Pi_{2}}\right) \\
- & \left(\frac{\left(\sigma_{L}(o)-\beta \hat{\rho}_{2}\right)\left(\Pi_{1}-2 \beta b(o) \hat{\rho}_{1} \rho_{1}\right)+b(o) \rho_{2} \Pi_{2}}{\Pi_{2}}\right) \frac{G_{o}(o, t)}{G(o, t)}, \\
\eta_{3}^{*}\left(w_{2}^{*}\right)= & \left(\frac{\beta^{2} \hat{\rho}_{1} \hat{\rho}_{3} \xi_{S}(o)}{\sigma_{1}(o) \Pi_{2}}\right)+\left(\frac{\beta \hat{\rho}_{3}\left(\xi_{L}(o)-v\right)}{\Pi_{2}}\right) \\
& +\left(\frac{\beta \hat{\rho}_{3} \Pi_{1}-b(o)\left(2 \beta^{2} \rho_{1} \hat{\rho}_{1} \hat{\rho}_{3}+\rho_{3} \Pi_{2}\right)}{\Pi_{2}}\right) \frac{G_{o}(o, t)}{G(o, t)},
\end{aligned}
$$




$$
\begin{aligned}
\eta_{4}^{*}= & \left(\frac{\beta^{2} \hat{\rho}_{1} \hat{\rho}_{4} \xi_{S}(o)}{\sigma_{1}(o) \Pi_{2}}\right)+\left(\frac{\beta \hat{\rho}_{4}\left(\xi_{L}(o)-v\right)}{\Pi_{2}}\right) \\
& +\left(\frac{\beta \hat{\rho}_{4} \Pi_{1}-2 b(o) \beta^{2} \rho_{1} \hat{\rho}_{1} \hat{\rho}_{3}}{\Pi_{2}}\right) \frac{G_{o}(o, t)}{G(o, t)} .
\end{aligned}
$$

where $\mathrm{G}$ is a unique bounded solution to

$$
G_{t}+\left(a(o)-d_{1}(o)\right) G_{o}+\frac{1}{2} b^{2}(o) G_{o o}-d_{2}(o) \frac{G_{o}^{2}}{G}+d_{3}(o) G=0,
$$

with terminal condition $G(0, T)=1$.

Proof. Since there exists a unique bounded solution to Equation (44), via Lemma 3 the derivative $G_{0}$ is bounded. If we set $V(x, y, o, t):=-x+G(o, t) y$, then it is sufficient to prove that the Markov saddle point $\kappa^{*}(x, y, o, t), \eta^{*}(x, y, o, t) \in \mathcal{A}_{t} \times \mathcal{M}$, and condition Equation (25) holds. Hence $G$ is bounded and $Y^{\eta^{*}}$ is a solution to the stochastic linear equation with bounded coefficient and it will imply that

$\mathbb{E}_{x, y, o, t}^{\eta}\left[\sup _{t \leq s \leq T}\left|G(O(s), s) Y^{\eta^{*}}(s)\right|\right]<+\infty$, for all $\eta \in \mathcal{M}$. To prove

$\left[\sup _{t \leq s \leq T}\left|X^{\kappa^{*}}(s)\right|\right]<+\infty$, we consider the fixed initial conditions $\left(x_{0}, y_{0}, o_{0}, t_{0}\right)$, and define the strategy $\kappa^{*}$

$$
\begin{aligned}
\hat{\kappa}^{*}= & -\left(X^{\kappa^{*}}(t)-x_{0}+2 y_{0} G\left(o_{0}, t_{0}\right)\left[\frac{\Pi_{0} \lambda_{1}(o)}{\sigma_{1}(0) \Pi_{2}}+\frac{\beta \hat{\rho}_{1} \lambda_{2}(o)}{\sigma_{1}(o)}\right.\right. \\
& \left.+\frac{\left(\beta \hat{\rho}_{1} \Pi_{1}-2 \rho_{1} b(o) \Pi_{0}\right)}{\sigma_{1}(o) \Pi_{2}} \frac{G_{o}(o, t)}{G(o, t)}\right]+2 y_{0} G\left(o_{0}, t_{0}\right)\left[\lambda_{2}(o)+\frac{\beta \hat{\rho}_{1} \lambda_{1}(o)}{\Pi_{2}}\right. \\
& \left.\left.+\left(\frac{\Pi_{1} \Pi_{2}+\beta^{2} \hat{\rho}_{1}^{2} \Pi_{1}-2 \beta b(o) \hat{\rho}_{1} \rho_{1} \Pi_{0}}{\Pi_{0} \Pi_{2}}\right) \frac{G_{o}(o, t)}{G(o, t)}\right]\right) .
\end{aligned}
$$

Now let us define:

$$
\begin{aligned}
\varphi_{1}(o, t):=-\left[\frac{\Pi_{0} \lambda_{1}(o)}{\sigma_{1}(0) \Pi_{2}}+\frac{\beta \hat{\rho}_{1} \lambda_{2}(o)}{\sigma_{1}(o)}+\frac{\left(\beta \hat{\rho}_{1} \Pi_{1}-2 \rho_{1} b(o) \Pi_{0}\right)}{\sigma_{1}(o) \Pi_{2}} \frac{G_{o}(o, t)}{G(o, t)}\right], \\
\varphi_{2}(o, t)=-\left[\lambda_{2}(o)+\frac{\beta \hat{\rho}_{1} \lambda_{1}(o)}{\Pi_{2}}\right. \\
\left.+\left(\frac{\Pi_{1} \Pi_{2}+\beta^{2} \hat{\rho}_{1}^{2} \Pi_{1}-2 \beta b(o) \hat{\rho}_{1} \rho_{1} \Pi_{0}}{\Pi_{0} \Pi_{2}}\right) \frac{G_{o}(o, t)}{G(o, t)}\right] .
\end{aligned}
$$

Furthermore, $\varphi_{1} \cdot\left(\xi_{S}\right), \varphi_{1} \cdot \sigma_{1}, \varphi_{2} \cdot\left(\xi_{L}-v\right)$ and $\varphi_{2} \cdot \sigma_{L}$ are bounded functions due to the fact that $\sum_{i=1}^{2} \lambda_{i}, \sum_{i=1}^{2}\left|\lambda_{i}^{2}\right|$, are bounded. Therefore the process $K(t):=X^{\kappa^{*}}(t)-x_{0}+2 y_{0} G\left(o_{0}, t_{0}\right)$ is a unique solution to the following equation

$$
\frac{\mathrm{d} K(t)}{K(t)}=\left(\begin{array}{c}
\varphi_{1}(O(t), t)\left(\xi_{S}(t)\right) \\
\varphi_{2}(O(t), t)\left(\xi_{L}(t)-v(t)\right)
\end{array}\right) \mathrm{d} t+\left(\begin{array}{c}
\varphi_{1}(O(t), t) \sigma_{1}(O(t)) \mathrm{d} W_{1}(t) \\
\varphi_{2}(O(t), t) \sigma_{L}(O(t)) \mathrm{d} W_{2}(t)
\end{array}\right) .
$$


This is a linear stochastic equation with bounded coefficients and satisfy $\mathbb{E}_{x_{0}, y_{0}, o_{0}, t_{0}}^{\eta}\left[\sup _{t \leq s \leq T}\left|X^{\kappa^{*}}(s)\right|\right]<+\infty$, for all $\eta \in \mathcal{M}$. This confirms admissibility of $\kappa^{*}=\left(w_{1}^{*}, w_{2}^{*}\right)$.

\section{Basel III CAR}

First step is to obtain the dynamics of the TRWAs with respect to the total asset portfolio of the bank.

Remark 3. Bank's Tier 1 and Tier 2 must be at least $8 \%$ of the total risk-weighted assets (TRWA's) and asset performance is a key albeit lagging indicator. The TRWA's are calculated in accordance with advanced internal measurement approach (AIRB) for the majority of group's credit risk exposures. Hence we describes the dynamics of TRWA's under risk regulation at time $t$, $a_{r w}(t)$ can be described by the stochastic differential equation

$$
\begin{aligned}
\frac{\mathrm{d} a_{r w}(t)}{a_{r w}(t)}= & \delta_{0}\left(1-w_{1}-w_{2}\right) \frac{\mathrm{d} M(t)}{M(t)}+\delta_{1}\left(w_{2}(t) \frac{\mathrm{d} L(t)}{L(t)}-w_{2}(t) \mathrm{d} \pi(t)\right) \\
& +\delta_{2} w_{1}(t) \frac{\mathrm{d} S(t)}{S(t)},
\end{aligned}
$$

where $\delta_{0}=0 . \delta_{1}$ and $\delta_{2}$ is estimated via AIRB and will lie between $0<\delta_{1}, \delta_{2}<1$ or this analysis purely as an example by setting $\delta_{1}=0.35$, $\delta_{2}=0.45$ and simplifying, we obtain

$$
\begin{aligned}
& \frac{\mathrm{d} a_{r w}(t)}{a_{r w}(t)}=\left[0.35 w_{2}(t)\left(r(t)+\xi_{L}(o)-v\right)+0.45 w_{1}(t)\left(r(t)+\xi_{S}(o)\right)\right] \mathrm{d} t \\
& +0.45 w_{1}(t) \sigma_{1}(o) \mathrm{d} W_{1}(t)+0.35 w_{2}(t) \sigma_{L}(o) \mathrm{d} W_{2}(t)-0.35 w_{2}(t) \beta \mathrm{d} \tilde{W}(t) .
\end{aligned}
$$

Proposition 1. (Explicit Indigo Partial Differential Equation for the Basel III $C A R)$

Suppose that the dynamics of total bank capital $C(t)$ and total risk-weighted assets $a_{r w}(t)$ are described by Equation (18) and Equation (46), respectively. Then the dynamics of the Basel III capital adequacy ratio $\chi(t)$ of a bank satisfies the following $S D E$.

$$
\begin{aligned}
\frac{\mathrm{d} \chi(t)}{\chi(t)}= & {\left[\tilde{\varphi}_{1}-\tilde{\varphi}_{2}-\tilde{\varphi}_{3}\right] \mathrm{d} t-\left[\beta_{1} \mathrm{~d} W_{1}(t)+\beta_{2} \mathrm{~d} W_{2}(t)+\beta_{3} \mathrm{~d} W_{3}(t)\right.} \\
& \left.+\beta_{4} \mathrm{~d} W_{4}(t)-\beta_{5} \mathrm{~d} \hat{W}(t)\right],
\end{aligned}
$$

where

$$
\begin{gathered}
\tilde{\varphi}_{1}=\tilde{\varphi}_{2} r(t)+w^{\top}(t) \Psi \eta, \quad \tilde{\varphi}_{2}=\rho X(t), \\
\tilde{\varphi}_{3}=\left\{\left[0.35 w_{2}(t)\left(r(t)+\xi_{L}(o)-v\right)+0.45 w_{1}(t)\left(r(t)+\xi_{S}(o)\right)\right]\right. \\
-\left[(0.45)^{2} w_{1}^{2}(t) \sigma_{1}^{2}(o)+(0.35)^{2} w_{2}^{2}(t) \beta^{2} \hat{\rho}_{1}^{2}\right. \\
\left.-2(0.45)(0.35) w_{1}(t) w_{2}(t) \beta \sigma_{1}(o) \hat{\rho}_{1}\right] \\
+(0.35)^{2} w_{2}^{2}(t)\left(\sigma_{L}(o)-\beta \hat{\rho}_{2}\right) \\
\left.+(0.35)^{2} w_{2}^{2}(t) \beta^{2} \hat{\rho}_{3}^{2}+(0.35)^{2} w_{2}^{2}(t) \beta^{2} \hat{\rho}_{4}^{2}\right\}
\end{gathered}
$$




$$
\begin{gathered}
\beta_{1}=0.45 w_{1}(t) \sigma_{1}(o)-0.35 w_{2}(t) \beta \hat{\rho}_{1}, \beta_{2}=0.35 w_{2}(t) \sigma_{L}(o)-0.35 w_{2}(t) \beta \hat{\rho}_{2}, \\
\beta_{3}=\beta_{4}-0.35 w_{2}(t) \beta \hat{\rho}_{3}, \beta_{4}=-0.35 w_{2}(t) \beta \hat{\rho}_{4}, \beta_{5}=w^{\top}(t) \Psi .
\end{gathered}
$$

Proof. In this proof we derive Equation (47) using the general ItÓ's formula. Let $f\left(a_{r w}(t)\right)=\left(a_{r w}\right)^{-1}$.

$$
\begin{aligned}
\mathrm{d} f\left(a_{r w}(t)\right)=\frac{\partial f(t)}{\partial t} \mathrm{~d} t+\frac{\partial f\left(a_{r w}(t)\right)}{\partial a_{r w}} \mathrm{~d} a_{r w}+\frac{1}{2} \frac{\partial^{2}}{\partial^{2} a_{r w}} f\left(a_{r w}(t)\right)\left(\mathrm{d} a_{r w}\right)^{2} \\
=0 \mathrm{~d} t-\frac{\mathrm{d} a_{r w}(t)}{a_{r w}^{2}(t)}+\frac{\left[\mathrm{d} a_{r w}(t)\right]^{2}}{a_{r w}^{3}(t)}-\frac{1}{a_{r w}(t)}\left\{\left[0.35 w_{2}(t)\left(r(t)+\xi_{L}(o)-v\right)\right.\right. \\
\left.+0.45 w_{1}(t)\left(r(t)+\xi_{S}(o)\right)\right] \mathrm{d} t+0.45 w_{1}(t) \sigma_{1}(o) \mathrm{d} W_{1}(t) \\
+0.35 w_{2}(t) \sigma_{L}(o) \mathrm{d} W_{2}(t)-0.35 w_{2}(t) \beta\left(\hat{\rho}_{1} \mathrm{~d} W_{1}(t)+\hat{\rho}_{2} \mathrm{~d} W_{2}(t)\right. \\
\left.\left.+\hat{\rho}_{3} \mathrm{~d} W_{3}(t)+\hat{\rho}_{4} \mathrm{~d} W_{4}(t)\right)\right\}+\frac{1}{a_{r w}(t)}\left[(0.45)^{2} w_{1}^{2}(t) \sigma_{1}^{2}(o)\right. \\
+(0.35)^{2} w_{2}^{2}(t) \beta^{2} \hat{\rho}_{1}^{2}-2(0.45)(0.35) w_{1}(t) w_{2}(t) \sigma_{1}(o) \beta \hat{\rho}_{1} \\
+(0.35)^{2} w_{2}^{2}(t)\left(\sigma_{L}(o)-\beta \hat{\rho}_{2}\right)+(0.35)^{2} w_{2}^{2}(t) \beta^{2} \hat{\rho}_{3}^{2} \\
\left.+(0.35)^{2} w_{2}^{2}(t) \beta^{2} \hat{\rho}_{4}^{2}\right] \mathrm{d} t .
\end{aligned}
$$

Through algebraic manipulation and re-arranging the drift, diffusion and jump part of $\mathrm{d} f\left(a_{r w}(t)\right)$. We obtain:

$$
\begin{aligned}
\mathrm{d} f\left(a_{r w}(t)\right)=- & \frac{1}{a_{r w}(t)}\left\{\left[0.35 w_{2}(t)\left(r(t)+\xi_{L}(o)-v\right)+0.45 w_{1}(t)\left(r(t)+\xi_{S}(o)\right)\right]\right. \\
- & {\left[(0.45)^{2} w_{1}^{2}(t) \sigma_{1}^{2}(o)+(0.35)^{2} w_{2}^{2}(t) \beta^{2} \hat{\rho}_{1}^{2}\right.} \\
- & 2(0.45)(0.35) w_{1}(t) w_{2}(t) \sigma_{1}(o) \beta \hat{\rho}_{1}+(0.35)^{2} w_{2}^{2}(t)\left(\sigma_{L}(o)-\beta \hat{\rho}_{2}\right)^{2} \\
& \left.\left.+(0.35)^{2} w_{2}^{2}(t) \beta^{2} \hat{\rho}_{3}^{2}+(0.35)^{2} w_{2}^{2}(t) \beta^{2} \hat{\rho}_{4}^{2}\right]\right\} \mathrm{d} t \\
& -\frac{1}{a_{r w}(t)}\left\{\left(0.45 w_{1}(t) \sigma_{1}(o)-0.35 w_{2}(t) \beta \hat{\rho}_{1}\right) \mathrm{d} W_{1}(t)\right. \\
& +\left(0.35 w_{2}(t) \sigma_{L}(o)-0.35 w_{2}(t) \beta \hat{\rho}_{2}\right) \mathrm{d} W_{2}(t) \\
& \left.-0.35 w_{2}(t) \beta \hat{\rho}_{3} \mathrm{~d} W_{3}(t)-0.35 w_{2}(t) \beta \hat{\rho}_{4} \mathrm{~d} W(t)\right\} .
\end{aligned}
$$

Then the CAR is expressed as $\chi(t)=\frac{C(t)}{a_{r w}(t)}=C(t) f\left(a_{r w}(t)\right)$. To find an expression for $\mathrm{d} Y(t)=\mathrm{d}\left(f\left(a_{r w}(t)\right) C(t)\right)$, we apply ItÓ's product rule to $\chi(t)$. As a result, we have

$$
\begin{aligned}
& \mathrm{d} \chi(t)=f\left(a_{r w}(t-)\right) \mathrm{d} C(t)+C(t) \mathrm{d} f\left(a_{r w}(t-)\right)+\mathrm{d}\left[f\left(a_{r w}\right), C\right](t) \\
&= f\left(a_{r w}(t-)\right)\left(C(t)\left[\left(r(t)+w^{\top}(t) \Psi \vartheta\right) \mathrm{d} t+w^{\top}(t) \Psi \mathrm{d} \hat{W}(t)\right]-\rho X(t) \mathrm{d} t\right) \\
&-\frac{C(t)}{a_{r w}(t-)}\left\{\left[0.35 w_{2}(t)\left(r(t)+\xi_{L}(o)-v\right)+0.45 w_{1}(t)\left(r(t)+\xi_{S}(o)\right)\right]\right. \\
&-\left[(0.45)^{2} w_{1}^{2}(t) \sigma_{1}^{2}(o)+(0.35)^{2} w_{2}^{2}(t) \beta^{2} \hat{\rho}_{1}^{2}\right. \\
&-2(0.45)(0.35) w_{1}(t) w_{2}(t) \sigma_{1}(o) \beta \hat{\rho}_{1}-\left[(0.35)^{2} w_{2}^{2}(t)\left(\sigma_{L}(o)-\beta \hat{\rho}_{2}\right)^{2}\right.
\end{aligned}
$$




$$
\begin{aligned}
& \left.\left.+(0.35)^{2} w_{2}^{2}(t) \beta^{2} \hat{\rho}_{3}^{2}-(0.35)^{2} w_{2}^{2}(t) \beta^{2} \hat{\rho}_{4}^{2}\right]\right\} \mathrm{d} t \\
& -\frac{C(t)}{a_{r w}(t)}\left\{\left(0.45 w_{1}(t) \sigma_{1}(o)-0.35 w_{2}(t) \beta \hat{\rho}_{1}\right) \mathrm{d} W_{1}(t)\right. \\
& +\left(0.35 w_{2}(t) \sigma_{L}(o)-0.35 w_{2}(t) \beta \hat{\rho}_{2}\right) \mathrm{d} W_{2}(t) \\
& \left.-0.35 w_{2}(t) \beta \hat{\rho}_{3} \mathrm{~d} W_{3}(t)-0.35 w_{2}(t) \beta \hat{\rho}_{4} \mathrm{~d} W_{4}(t)\right\} .
\end{aligned}
$$

Grouping the drift and diffusion rems of $\mathrm{d} \chi(t)$ results in

$$
\begin{aligned}
\frac{\mathrm{d} \chi(t)}{\chi(t)}= & {\left[\tilde{\varphi}_{1}-\tilde{\varphi}_{2}-\tilde{\varphi}_{3}\right] \mathrm{d} t-\left[\beta_{1} \mathrm{~d} W_{1}(t)+\beta_{2} \mathrm{~d} W_{2}(t)+\beta_{3} \mathrm{~d} W_{3}(t)\right.} \\
& \left.+\beta_{4} \mathrm{~d} W_{4}(t)-\beta_{5} \mathrm{~d} \hat{W}(t)\right],
\end{aligned}
$$

where we have introduced and defined $\tilde{\varphi}_{1}, \tilde{\varphi}_{2}, \tilde{\varphi}_{3}, \beta_{1}, \beta_{2}, \beta_{3}, \beta_{4}$ and $\beta_{5}$ in the formulation of this proposition.

\section{Relation to Mean-Variance Optimization}

Since the motivation of our objective function comes from mean-variation optimization literature, we compare our results with such mean-variance optimization. In order to consider the mean-variance problem we consider the following functional

$$
\mathcal{I}^{\kappa^{*}}(x, o, 0):=\mathbb{E}_{x, o, 0} X^{\kappa}(T)-\gamma \mathbb{D}_{x, o, 0}^{2} X^{\kappa}(T), \gamma>0,
$$

where $\gamma$ is the bank's risk aversion coefficient and $\mathbb{D}_{x, o, 0}^{2} X^{\kappa}(T):=\mathbb{E}_{x, o, 0}\left[X^{\kappa}(T)-\mathbb{E}_{x, o, 0} X^{\kappa}(T)\right]^{2}$. The aim of the banker is to maximize $\mathcal{I}^{\kappa^{*}}(x, o, 0)$ with respect to $\kappa=\left(w_{1}, w_{2}\right) \in \mathcal{A}_{0}$. Following [32], we define

$$
\begin{aligned}
\sup _{\kappa \in \mathcal{A}_{0}} \mathcal{I}^{\kappa}(x, o, 0) & =\sup _{\kappa \in \mathcal{A}_{0}}\left\{\mathbb{E}_{x, o, 0} X^{\kappa}(T)-\gamma \mathbb{E}_{x, o, 0}\left[X^{\kappa}(T)-\mathbb{E}_{x, o, 0} X^{\kappa}(T)\right]^{2}\right\} \\
& =\sup _{A \in \mathbb{R}} \sup _{\kappa \in \mathcal{A}_{t}}\left\{A-\gamma \mathbb{E}_{x, o, 0}\left[X^{\kappa}(T)-A\right]^{2}\right\},
\end{aligned}
$$

where $\tilde{\mathcal{A}}_{t}=\left\{\kappa \in \mathcal{A}_{t}: \mathbb{E}_{x, o, 0} X^{\kappa}(T)=A, A \in \mathbb{R}\right\}$. Hence we have replaced the unconstrained mean-variance optimization problem with a constrained maximization of quadratic objective and using Lagrange method we obtain the minimization functional as:

$$
\begin{aligned}
I^{\kappa(l)}(x, o, t) & :=\mathbb{E}_{x, o, t}\left[X^{\kappa(l)}(T)-A\right]^{2}-2 l \mathbb{E}_{x, o, t} X^{\kappa(l)}(T) \\
& =\mathbb{E}_{x, o, t}\left[X^{\kappa(l)}(T)-(A+l)\right]^{2}-2 A l-l^{2}
\end{aligned}
$$

to determine the solution $\kappa^{*}(l)$ over a class of admissible controls $\tilde{\mathcal{A}}_{t}$ and we find $l^{*}$ such that $\mathbb{E}_{x, o, 0} X^{\kappa^{*}\left(l^{*}\right)}(T)=A$. Applying Theorem 4.1 of [21] we have the optimal strategy for functional Equation (51) as:

$$
\begin{aligned}
\kappa^{*}(l, x, o, t)= & -\left(x-(A+l)\left[\frac{\Pi_{0} \lambda_{1}(o)}{\sigma_{1}(o) \Pi_{2}}+\frac{\beta \hat{\rho}_{1} \lambda_{2}(o)}{\sigma_{1}(o)}\right.\right. \\
& \left.+\frac{\left(\beta \hat{\rho}_{1} \Pi_{1}-2 \rho_{1} b(o) \Pi_{0}\right)}{\sigma_{1}(o) \Pi_{2}} \frac{H_{o}(o, t)}{H(o, t)}\right]-(A+l)\left[\lambda_{2}(o)+\frac{\beta \hat{\rho}_{1} \lambda_{1}(o)}{\Pi_{2}}\right. \\
& \left.\left.+\left(\frac{\Pi_{1} \Pi_{2}+\beta^{2} \hat{\rho}_{1}^{2} \Pi_{1}-2 \beta b(o) \hat{\rho}_{1} \rho_{1} \Pi_{0}}{\Pi_{0} \Pi_{2}}\right) \frac{H_{o}(o, t)}{H(o, t)}\right]\right)
\end{aligned}
$$


where $H$ satisfies

$$
H_{t}+\left(a(o)-d_{1}(o)\right) H_{o}+\frac{1}{2} b^{2}(o) H_{o o}-d_{2}(o) \frac{H_{o}^{2}}{H}-d_{3}(o) H=0,
$$

together with the terminal condition $H(o, T)=1$. Noting that $G=-\frac{1}{H}$, is a solution to

$$
G_{t}+\left(a(o)-d_{1}(o)\right) G_{o}+\frac{1}{2} b^{2}(o) G_{o o}-d_{2}(o) \frac{G_{o}^{2}}{G}+d_{3}(o) G=0,
$$

where $G(o, T)=1$. In addition, we have

$$
\begin{aligned}
\kappa^{*}(l, x, o, t) & -\left(x-(A+l)\left[\frac{\Pi_{0} \lambda_{1}(o)}{\sigma_{1}(o) \Pi_{2}}+\frac{\beta \hat{\rho}_{1} \lambda_{2}(o)}{\sigma_{1}(o)}\right.\right. \\
& \left.+\frac{\left(\beta \hat{\rho}_{1} \Pi_{1}-2 \rho_{1} b(o) \Pi_{0}\right)}{\sigma_{1}(o) \Pi_{2}} \frac{H_{o}(o, t)}{H(o, t)}\right]-(A+l)\left[\lambda_{2}(o)+\frac{\beta \hat{\rho}_{1} \lambda_{1}(o)}{\Pi_{2}}\right. \\
& \left.\left.+\left(\frac{\Pi_{1} \Pi_{2}+\beta^{2} \hat{\rho}_{1}^{2} \Pi_{1}-2 \beta b(o) \hat{\rho}_{1} \rho_{1} \Pi_{0}}{\Pi_{0} \Pi_{2}}\right) \frac{H_{o}(o, t)}{H(o, t)}\right]\right),
\end{aligned}
$$

confirming that the quadratic optimization is consistent with monotone optimization with suitable $A$ and $l$.

In order to identify $l^{*}$, we recall $\mathbb{E}_{x, o, 0} X^{\kappa^{*}\left(l^{*}\right)}(T)=A$, and define

$$
P(t):=X^{\kappa^{*}\left(l^{*}\right)}(t)-\left(A+l^{*}\right) .
$$

Since $\varphi_{1} \cdot\left(\xi_{S}\right), \varphi_{1} \cdot \sigma_{1}, \varphi_{2} \cdot\left(\xi_{L}-v\right)$ and $\varphi_{2} \cdot \sigma_{L}$, are bounded functions and $P(t)$ is a solution to the stochastic linear equation with bounded coefficients, we obtain

$$
\frac{\mathrm{d} P(t)}{P(t)}=\left(\begin{array}{c}
\varphi_{1}(O(t), t)\left(\xi_{S}(O(t))\right) \\
\varphi_{2}(O(t), t)\left(\xi_{L}(O(t))-v(t)\right)
\end{array}\right) \mathrm{d} t+\left(\begin{array}{c}
\varphi_{1}(O(t), t) \sigma_{1}(O(t)) \mathrm{d} W_{1}(t) \\
\varphi_{2}(O(t), t) \sigma_{L}(O(t)) \mathrm{d} W_{2}(t)
\end{array}\right) .
$$

This implies that $P(t)=\left(x-\left(A+l^{*}\right)\right) R(t)$ where $R(t)$ is given by

$$
\begin{aligned}
R(t):= & \exp \left\{\int_{0}^{t} \varphi_{1}(O(s), s) \xi_{S}(O(s))-\frac{1}{2} \varphi_{1}^{2}(O(s), s) \sigma_{1}^{2}(O(s)) \mathrm{d} s\right. \\
& +\varphi_{2}(O(s), s)\left(\xi_{L}(O(s))-v(s)\right)-\frac{1}{2} \varphi_{2}^{2}(O(s), s) \sigma_{L}^{2}(O(s)) \mathrm{d} s \\
& \left.+\int_{0}^{t} \varphi_{1}(O(s), s) \sigma_{1}(O(s)) \mathrm{d} W_{1}(s)+\int_{0}^{t} \varphi_{2}(O(s), s) \sigma_{L}(O(s)) \mathrm{d} W_{2}(s)\right\} .
\end{aligned}
$$

Using Equation (52) we have $X^{\kappa^{*}\left(l^{*}\right)}(t)=(x-A) R(T)+A+l^{*}(1-R(T))$. This implies that

$$
l^{*}(A)=\frac{(A-x) \mathbb{E}_{o, 0} R(T)}{1-\mathbb{E}_{o, 0} R(T)}, \text { if } \mathbb{E}_{o, 0} R(T) \neq 1 .
$$

Finally, we note that

$$
A A-\gamma \mathbb{E}_{o, 0}\left[X^{\kappa^{\star}\left(l^{\star}\right)}(T)-A\right]^{2}=A-\gamma \mathbb{E}_{o, 0}\left[(x-A) R(T)+\gamma^{\star}(A)(1-R(T))\right]^{2}
$$


and the maximum over $A$ is attained as

$$
A^{*}=x+\frac{1}{2 \gamma \mathbb{E}_{o, 0} \Gamma^{2}(T)},
$$

where $\Gamma^{2}(T):=\frac{R(T)-\mathbb{E}_{x, o, 0} R(T)}{1-\mathbb{E}_{x, o, 0} R(T)}$. Substituting it into Equation (53) and Equation (54) we obtain

$$
A^{*}=x+\frac{1}{2 \gamma} \frac{\left(1-\mathbb{E}_{o, 0} R(T)\right)}{\mathbb{D}_{o, 0}^{2} R(T)} \text { and } l^{*}\left(A^{*}\right)=\frac{1}{2 \gamma} \frac{\left(1-\mathbb{E}_{o, 0} R(T)\right) \mathbb{E}_{o, 0} R(T)}{\mathbb{D}_{o, 0}^{2} R(T)} .
$$

Consideration Equation (52) we conclude that the banker's mean-variance optimal strategy is given by

$$
\begin{aligned}
\kappa^{*}(x, o, t)= & -\left[x-x_{0}-\left(\frac{1}{2 \gamma} \frac{1-\mathbb{E}_{o_{0}, 0} R(T)}{\mathbb{D}_{o_{0}, 0}^{2} R(T)}\right)\left(\frac{\Pi_{0} \lambda_{1}(o)}{\sigma_{1}(o) \Pi_{2}}+\frac{\beta \hat{\rho}_{1} \lambda_{2}(o)}{\sigma_{1}(o)}\right.\right. \\
& \left.+\frac{\left(\beta \hat{\rho}_{1} \Pi_{1}-2 \rho_{1} b(o) \Pi_{0}\right)}{\sigma_{1}(o) \Pi_{2}} \frac{G_{o}(o, t)}{G(o, t)}\right) \\
& -\left(\frac{1}{2 \gamma} \frac{1-\mathbb{E}_{o_{0}, 0} R(T)}{\mathbb{D}_{o_{0}, 0}^{2} R(T)}\right)\left(\lambda_{2}(o)+\frac{\beta \hat{\rho}_{1} \lambda_{1}(o)}{\Pi_{2}}\right. \\
& \left.\left.+\left(\frac{\Pi_{1} \Pi_{2}+\beta^{2} \hat{\rho}_{1}^{2} \Pi_{1}-2 \beta b(o) \hat{\rho}_{1} \rho_{1} \Pi_{0}}{\Pi_{0} \Pi_{2}}\right) \frac{G_{o}(o, t)}{G(o, t)}\right)\right] .
\end{aligned}
$$

The dynamics of the banker's monotone optimal strategy is given as:

$$
\begin{aligned}
\kappa^{*}(x, o, t)= & -\left[x-x_{0}+2 y_{0} G\left(o_{0}, 0\right)\left(\frac{\Pi_{0} \lambda_{1}(o)}{\sigma_{1}(o) \Pi_{2}}+\frac{\beta \hat{\rho}_{1} \lambda_{2}(o)}{\sigma_{1}(o)}\right.\right. \\
& \left.+\frac{\left(\beta \hat{\rho}_{1} \Pi_{1}-2 \rho_{1} b(o) \Pi_{0}\right)}{\sigma_{1}(o(t)) \Pi_{2}} \frac{G_{o}(o, t)}{G(o, t)}\right) \\
& +2 y_{0} G\left(o_{0}, 0\right)\left(\lambda_{2}(o)+\frac{\beta \hat{\rho}_{1} \lambda_{1}(o)}{\Pi_{2}}\right. \\
& \left.\left.+\left(\frac{\Pi_{1} \Pi_{2}+\beta^{2} \hat{\rho}_{1}^{2} \Pi_{1}-2 \beta b(o) \hat{\rho}_{1} \rho_{1} \Pi_{0}}{\Pi_{0} \Pi_{2}}\right) \frac{G_{o}(o, t)}{G(o, t)}\right)\right] .
\end{aligned}
$$

Hence, we quantify the banker's risk-aversion factor as

$$
\gamma=\frac{1-\mathbb{E}_{o_{0}, 0} R(T)}{4 y_{0} G\left(o_{0}, 0\right) \mathbb{D}_{o_{0}, 0}^{2} R(T)} .
$$

\section{Conclusions}

In this study we examined continuous time optimization incorporating inflation, assuming that the preference criterion is based on a modification of a monotone mean-variance functional introduced by Maccheroni et al. [27]. There are two risky assets available to invest, and the compositional changes in bankers' asset portfolio allocation between stock index fund and the loan portfolio is due to the influence of the state variable (inflation). We demonstrate that in the presence of 
anticipated inflation, the banker's capital in Tier I and Tier II can be reduced and radically alters the banker's optimal holdings between the myopically optimal portfolio and intertemporal hedging portfolio. Specifically, it is assumed that banks allocate their assets such that they are able to repay all of their debts even when the maximum loss on both types of assets materializes. Our model suggests that, changes in inflation will contribute towards the deterioration in banks' balance sheets, a slowdown in lending or investment strategies. As these changes affect the tightness of the Tier I and Tier II capital holdings due to Basel II CAR requirements, if banks asset allocation tilts toward stock index fund holdings due to a re-balancing of banks asset allocation, the supply of capital via loans to goods producers' decline. As a result, output decreases and deflation emerges. Our results confirm that the presence of inflation risk radically alters the banker's optimal holdings and the trade-off between holding a myopically optimal portfolio and intertemporal hedging demand is determined by the derivatives of marginal utility with respect to the state variable.

In addition, the model helps to capture the extent of deterring Tier I and Tier II bank's adequate capital in the presence of aggregate risk and will help to set up a risk management strategy via diversifying its investment portfolio. On the other hand, the bank may choose to use financial instruments to mitigate these aggregated risks.

Future research could apply the maximum principle and backward Stochastic Differential Equations (BSDE) method over our method (dynamic programing principle and Hamilton-Jacobi-Bellman equations). Similarly, one could apply HJBI dynamic programming principle by capturing the banker's provision capital risk process via a jump-diffusion process, as oppose to the diffusion process applied in the study. However, in doing one may not able to obtain a closedform solution.

\section{Conflicts of Interest}

The author declares no conflicts of interest regarding the publication of this paper.

\section{References}

[1] Basel Committee on Banking Supervision (2004) International Convergence of Capital Measurement and Capital Standards: A Revised Framework. Bank for International Settlements, Basel. http://www.bis.org/publ/bcbs107.pdf

[2] Basel Committee on Banking Supervision (2011) Basel III: A Global Regulatory Framework for More Resilient Banks and Banking Systems-Revised Version June 2011. Bank for International Settlements. http://bis.org/publ/bcbs189.htm

[3] Ferguson, R. (2003) Remarks at the Risk Management Association's Conference on Capital Management, Washington, DC, April 9.

[4] Berger, A.N. and Udell, G.F. (1994) Did Risk-Based Capital Allocate Bank Credit and Cause a Credit Crunch in the United States? Journal of Money, Credit and Banking, 26, 585-628. https://doi.org/10.2307/2077994

[5] Jones, D. (2000) Emerging Problems with the Basel Capital Accord: Regulatory 
Capital Arbitrage and Related Issues. Journal of Banking and Finance, 24, 35-58. https://doi.org/10.1016/S0378-4266(99)00052-7

[6] Brinkmann, E.J. and Horvitz, P. (1995) Risk-Based Capital Standards and the Credit Crunch. Journal of Money, Credit and Banking, 27, 848-863. https://doi.org/10.2307/2077755

[7] Furfine, C. (2001) Bank Portfolio Allocation: The Impact of Capital Requirements, Regulatory Monitoring, and Economic Conditions. Journal of Financial Services Research, 20, 33-56.

[8] Hall, B. (1993) How Has the Basel Accord Affected Bank Portfolios? Journal of Japanese and International Economics, 7, 408-440.

https://doi.org/10.1006/jjie.1993.1024

[9] Haubrich, J.G. and Wachtel, P. (1993) Capital Requirements and Shifts in Commercial Bank Portfolios. Economic Review of Federal Research Bank of Cleveland, 3, 2-15.

[10] Thakor, A.V. (1996) Capital Requirement Monetary Policy and Aggregated Bank Lending. Journal of Finance, 51, 279-324. https://doi.org/10.1111/j.1540-6261.1996.tb05210.x

[11] Jacques, K.T. and Nigro, P. (1997) Risk-Based Capital, Portfolio Risk, and Bank Capital: A Simultaneous Equations Approach. Journal of Economics and Business, 49, 533-547. https://doi.org/10.1016/S0148-6195(97)00038-6

[12] Jacques, K.T. (2008) Capital Shocks, Bank Asset Allocation, and the Revised Basel Accord. Review of Financial Economics, 17, 79-91. https://doi.org/10.1016/j.rfe.2007.03.003

[13] Keeton, W.R. (1994) Causes of the Recent Increase in Bank Security Holdings. Economic Review, 78, 45-57.

[14] Borio, C., Furfine, C. and Lowe, P. (2001) Procyclicality of the Financial System and Financial Stability: Issues and Policy Options. Bank for International Settlements Conference Marrying the Macro-and-Micro Prudential Dimensions of Financial Stability, 1-57.

[15] Deelstra, G., Grasselli, M. and Koehl, P.F. (2000) Optimal Investment Strategies in a CIR Framework. Journal of Applied Probability, 37, 1-12. https://doi.org/10.1239/jap/1014843074

[16] Fouche, C.H., Mukuddem-Petersen, J. and Petersen, M.A. (2005) Continuous-Time Stochastic Modelling of Capital Adequacy Ratios for Banks. Applied Stochastic Models in Business and Industry, 22, 41-71.

[17] Lowe, P. (2002) Credit Risk Measurement and Procyclicality. Bank for International Settlements Working Paper, No. 116, 1-174.

[18] Mataramvura, S. and Øksendal, B. (2008) Risk Minimizing Portfolios and HJBI Equations for Stochastic Differential Games. Stochastic, 80, 317-337. https://doi.org/10.1080/17442500701655408

[19] Witbooi, P.J., Van Schalkwyk, G.J. and Muller, G.E. (2011) An Optimal Investment Strategy in Bank Management. Mathematical Methods in Applied Science, 34, 1606-1617. https://doi.org/10.1002/mma.1467

[20] Perera, R.S. (2015) Dynamics Asset Allocation for a Bank under CRRA and HARA Framework. International Journal of Financial Engineering, 2, Article ID: 1550031.

[21] Perera, R.S. (2017) An Optimal Investment and Risk Control Policy for a Bank Under Exponential Utility. Stochastic Models, 33, 343-375.

https://doi.org/10.1080/15326349.2017.1300775 
[22] Perera, R.S. and Sato, K. (2018) Optimal Asset Allocation for a Bank under Risk Control. International Journal of Financial Engineering, 5, Article ID: 1850022.

[23] Monroe, M.F. (2010) Basel III Redefines Capital, American Bankers Association. ABA Banking Journal, 102, 33-35.

[24] Mukuuddem-Petersen, J. and Petersen, M.A. (2008) Optimizing Asset and Capital Adequacy Management in Banking. Journal of Optimization and Theory Applications, 137, 205-230. https://doi.org/10.1007/s10957-007-9322-X

[25] Von Thadden, E.L. (2004) Bank Capital Adequacy Regulation under the New Basel Accord. Journal of Financial Intermediation, 13, 90-95. https://doi.org/10.1016/j.jfi.2003.04.002

[26] Markowitz, H. (1952) Portfolio Selection. Journal of Finance, 7, 77-91.

[27] Maccheroni, F., Marinacci, M., Rustichini, A. and Taboga, M. (2009) Portfolio Selection with Monotone Mean-Variance Preferences. Mathematical Finance, 19, 487-521. https://doi.org/10.1111/j.1467-9965.2009.00376.x

[28] Bordigoni, G., Matoussi, A. and Schweizer, M. (2007) A Stochastic Control Approach to a Robust Utility Maximization Problem. Stochastic Analysis and Application, The Abel Symposium, Springer, Berlin, 125-151.

[29] Hernández, D. and Schied, A. (2007) A Control Approach to Robust Utility Maximization with Logarithmic Utility and Time-Consistent Penalties. Stochastic Process and Applications, 117, 980-1000. https://doi.org/10.1016/j.spa.2006.11.005

[30] Øksendal, B. and Sulem, A. (2014) Forward-Backward Stochastic Differential Games and Stochastic Control under Model Uncertainty. Journal of Optimization and Theory Applications, 161, 22-55. https://doi.org/10.1007/s10957-012-0166-7

[31] Trybula, J. and Dawisza, D. (2012) Continuous Time Portfolio Choice under Monotone Preferences with Quadratic Penalty-Stochastic Factor Case. http://arxiv.org/pdf/1403.3212.pdf

[32] Zawiza, D. (2012) Target Achieving Portfolio under Model Specification: Quadratic Optimization Framework. Applicationes Mathematicae, 39, 425-443. https://doi.org/10.4064/am39-4-3

[33] Zariphopoulou, T. (2001) A Solution Approach to Valuation with Unhegeable Risks. Finance and Stochastic, 5, 61-82. https://doi.org/10.1007/PL00000040

[34] Greenbaum, S.I. and Thakor, A.V. (2010) Contemporary Financial Intermediation. 2nd Edition, Advanced Finance Series, Academic Press, New York.

[35] Merton, R.C. (1974) On the Pricing of Corporate Debt: The Risk Structure of Interest Rates. Journal of Finance, 29, 449-470.

[36] Fleming, W. and Soner, H.M. (2006) Controlled Markov Processes and Viscosity Solutions. 2nd Edition, Springer, New York.

[37] Zawiza, D. (2010) Robust Portfolio Selection under Exponential Preferences. Applicationes Mathematicae, 37, 215-230. https://doi.org/10.4064/am37-2-6

[38] Korn, R. and Korn, E. (2001) Option Pricing and Portfolio Optimization. Graduate Studies in Mathematics, Vol. 31.

[39] Zhou, X.Y. and Li, D. (2000) Continuous Time Mean-Variance Portfolio Selection: A Stochastic LQ Framework. Journal of Applied Mathematics and Optimization, 42, 19-33.

[40] Heath, D. and Schweizer, M. (2000) Martingale versus PDEs in Finance: An Equivalence Result with Examples. Journal of Applied Probability, 37, 947-957.

https://doi.org/10.1239/jap/1014843075 
[41] Pham, H. (2009) Continuous-Time Stochastic Control and Optimization with Financial Applications, Stochastic Modelling and Applied Probability. Springer-Verlag, Berlin. https://doi.org/10.1007/978-3-540-89500-8 